\title{
MATHEMATICAL ANALYSIS OF THE ROLE OF DETECTION RATE IN THE DYNAMICAL SPREAD OF HIV-TB CO-INFECTION
}

\author{
I.A.Olopade ${ }^{1}$, S.O. Adewale ${ }^{2}$, I.T. Mohammed ${ }^{3}$, S.O, Ajao ${ }^{4}$ and O.T. Oyedemi ${ }^{5}$ \\ Ladoke Akintola University of Technology, (LAUTECH), Ogbomoso, P.M.B. 4000, Ogbomoso, Oyo State, \\ Nigeria. \\ isaac.sola.ia@gmail.com \\ Ladoke Akintola University of Technology, (LAUTECH), Ogbomoso, P.M.B. 4000, Ogbomoso, Oyo State, \\ Nigeria. \\ soadewale@lautech.edu.ng \\ Osun State Polytechnic Iree, P.M.B. 301,Iree,Osun State, Nigeria. \\ iskiludayat@gmail.com \\ Ladoke Akintola University of Technology, (LAUTECH), Ogbomoso, P.M.B. 4000, Ogbomoso, Oyo State, \\ Nigeria. \\ goajao@yahoo.co.uk \\ Ladoke Akintola University of Technology, (LAUTECH), Ogbomoso, P.M.B. 4000, Ogbomoso, Oyo State, \\ Nigeria. \\ oyedemioyebimpe@gmail.com
}

\begin{abstract}
Human Immunodeficiency Virus (HIV) co-existing with Tuberculosis (TB) in individuals remains a major global health challenges, with an estimated 1.4 million patients worldwide. These two diseases are enormous public health burden, and unfortunately, not much has been done in terms of modeling the dynamics of HIV-TB coinfection at a population level. We formulated new fifteen (15) compartmental models to gain more insight into the effect of treatment and detection of infected undetected individuals on the dynamical spread of HIV- TB co-infection.
\end{abstract}

Sub models of HIV and TB only were considered first, followed by the full HIV-TB co-infection model. Existence and uniqueness of HIV and TB only model were analyzed quantitatively, and we shown that HIV model only and TB only model have solutions, moreover, the solutions are unique. Stability of HIV model only, TB model only and full model of HIV-TB co-infection were analyzed for the existence of the disease free and endemic equilibrium points. Basic reproduction number $\left(R_{0}\right)$ was analyzed, using next generation matrix method (NGM), and it has been shown that the disease free equilibrium point is locally asymptotically stable whenever $R_{0}<1$ and unstable whenever this threshold exceeds unity. i.e. $R_{0}>1$, Numerical simulation was carried out by maple software using differential transformation method, to show the effect of treatment and detection of infected undetected individuals on the dynamical spread of HIV-TB co-infection.

Significantly, all the results obtained from this research show the importance of treatment and detection of infected undetected individuals on the dynamical spread of HIV-TB co-infection. Detection rate of infected undetected individuals reduce the spread of HIV-TB co-infections.

KEYWORDS: HIV; TB, Stability; Reproduction Number; Critical points; Co-infection

CORRESPONDING AUTHOR: Adewale S.O.

\section{Council for Innovative Research}

Peer Review Research Publishing System

Journal: JOURNAL OF ADVANCES IN MATHEMATICS

Vol. 11, No. 10

www.cirworld.com, editor@cirworld.com 


\subsection{INTRODUCTION}

1.1 Human Immunodeficiency Virus (HIV) is the etiological agent that causes acquired Immunodeficiency Syndrome (AIDS) when left untreated. HIV weakens immune system by attacks many blood cells, it wrecks T-cell in the blood by destroying and decreasing their number leading to decline in body's immunity to fight infection [28]. The term AIDS refers to only the last stage of the HIV infection after which death occurs. The HIV epidemic is posing threat to the world both developed and developing nations as HIV has infected millions of people all over the world without any restriction of nationality, religion etc[25]. HIV infection in adults (75-80 percent) has been transmitted to one partner through unprotected sexual intercourse when the other partner is infected with HIV. The sexual transmission is believed globally responsible for the majority of new HIV infections [22, 24].

There are multiple modes of HIV transmission including Sexual intercourse, sharing needles and sharp objects with HIV infected persons, or via HIV-contaminated blood transfusions [30]. Infants may acquire HIV at birth or through breast feeding if the mother is HIV positive, this type of transmission is known as vertical transmission [8]. Mother to child transmission (vertical infection), accounts for more than 90 percent of global infection to infants and children. As HIV infection progresses, immunity declines and patients tend to become more susceptible to common infections. The absolute T-helper cell $\left(\mathrm{CD4}^{+}\right)$count or percentage is used most often to evaluate the progression of HIV infection and to help clinicians make treatment decisions. [18].

1.2 TUBERCULOSIS (TB) is an airborne infectious disease caused by a bacterium called a MYCOBICTERIUM TUBERCULOSIS (Tubercle bacilli).It typically affects lung and other parts of the body. TB can be acquired when an individual is exposed to tubercle bacilli produced by an infective individual. The risk of infection with the tubercle bacilli is directly related to the degree of exposure and less to genetic or other host factors. In those with HIV, the risk of developing active TB increases to nearly $18 \%$ per year [3,7]. TB is one of the major global cause of death, especially in developing countries. Even in many countries where its overall incidence is low, TB remains a problem; there has been an outbreak in the New York in the recent past [5], and incidence is currently rising in the United Kingdom ( UK) [16] . In 2004, TB caused an estimated 1.7 million deaths and 8.9 million new cases of infection [36], Worldwide, 9.15 million new infections occurred in the year 2006. In 2009, WHO estimated that there were 9.4 million incident cases of TB and 1.3 million deaths due to TB among HIV negative cases globally [35]. Over $95 \%$ of infections are in developing countries, where TB remains a dominant cause of morbidity and mortality [18].

Even today after the development of advanced screening, diagnostic and treatment method for the disease, it is shown that about one third of the world's population has been exposed and is infected with tuberculosis. [27]. anyone can become infected with T.B. bacteria, but people with HIV infectious disease suffers greater risk of getting infected with this disease. T.B. is the second leading cause of death among people with infectious disease after HIV [29].

TB infection occurs when droplet nuclei containing tubercle bacilli are inhaled into the lungs and deposited in the alveoli. It is spread when individuals with active TB disease cough, sneeze, sing, laugh or through interaction with infectious individual. [1] After a person becomes infected, the tuberculosis bacteria are controlled by the person's immune system and the infection becomes latent. The infection becomes active, when the bacteria spread out of control most especially when the tubercle bacilli overwhelm the immune system and break out of the tubercles in the alveoli and spread to the lungs and other parts of the body via bloodstream. [35]. If not treated active TB can be fatal. TB is curable disease by the implementation of antibiotics, which decrease the mortality of TB to a minimal level, for instance, a $70 \%$ reduction in TB related mortality was recorded in the United State of America (USA) between 1945-1955 [2]. Many doctors prefer to hospitalize the patient in order to observe him or her during treatment, the method is called Direct Observed Therapy Short Course (DOTS), huge success has been achieved by this method. [1].

\subsection{A BRIEF BACKGROUND ON HIV AND TB CO-INFECTIONS}

The synergetic effect produced by the interaction of mycobacterium tuberculosis (TB) and the human immune-deficiency syndrome (HIV) are well known [26, 19, 20,34], where each accelerates the progression of the other. For instance, since its emergence in the 1980s, the HIV/AIDS pandemic continues to play a major role in the resurgence of TB disease which has greatly increased the morbidity and mortality rate of the disease worldwide. While HIV infections increase the risk of many opportunistic infections, the interactions between HIV and several infectious disease agents have caused particular medical and public health concern. [26]. For adults co-infected with HIV and latent TB, the life time risk of developing active TB disease rises from an estimated $10 \%$ up to $50 \%$. This resulted in a parallel pandemic of HIV/AIDS and TB disease where HIV prevalence is high. [18]. Persons co-infected with TB and HIV may spread the disease not only to other HIV-infected persons, but also members of the general population who do not participate in any of the high risk behaviours associated with HIV. The largest increase in the number of TB cases has been in men aged 25-44. [33].

HIV fuels progression to active disease in people infected with TB [13]. Rate of recurrence of TB, both due to endogenous reactivation and exogenous re-infection, are increased among people infected with HIV, [18]. For instance, in many countries of eastern and southern Africa, the rates of TB notification have increased by five or more times as a result of HIV infection. Similarly, TB also fuels the progression of HIV infection in the patient to full blown AIDS after which death occurs. [37].

Currently, about $8 \%$ of global TB cases are linked to HIV infection, but this number is likely to increase in the future. For instance, the number of HIV positives in India is estimated to be 3.97 million cases, and almost $60 \%$ of the reported cases of AIDS had TB. 
The interaction of TB and HIV/AIDS is on the increase and these infections occur mostly in poor economically regions of the world. The immunodeficiency caused by HIV infection reactivates latent TB infection and accelerates the progression of newly acquired TB [15].

The enormous public health burden inflicted by these two deadly diseases necessitates the use of mathematical modeling to gain insights into the effects of treatment on the infected detected individuals and detection of infected undetected individuals on the dynamical spread of the disease.

\subsection{MATHEMATICAL MODEL FORMULATION}

A non linear mathematical model is formulated and analyzed to study the effect of detection of undetected individuals on the dynamical spread of HIV- TB co-infection.

In modeling the dynamics, the total homogeneously mixing population at time t, denoted by $\mathrm{N}(\mathrm{t})$, is divided into (15) fifteen mutually-exclusive compartments of Susceptible $(\mathrm{S}(\mathrm{t}))$ individuals, Latently HIV $\left(L_{H}(t)\right)$ individuals, HIV Undetected $\left(H_{U}(t)\right)$ individuals, HIV Detected $\left(H_{D}(t)\right)$ individuals, Treated HIV $\left(H_{W}(t)\right)$ individuals, Latently TB induced HIV $\left(L_{T H}(t)\right)$ individuals, Active TB Induced HIV $\left(A_{T H}(t)\right)$ individuals, Latently HIV induced TB $\left(L_{H T}(t)\right)$ individuals, Active HIV induced TB $\left(A_{H T}(t)\right)$ individuals, Latent TB $\left(L_{T}(t)\right)$ individuals, TB Undetected $\left(T_{U}(t)\right)$ individuals, TB Detected $\left(T_{D}(t)\right)$ individuals, Failed Treatment TB $\left(F_{T}(t)\right)$ individuals, Recovered TB $\left(R_{T}(t)\right)$ individuals, Recovered TB induced HIV $\left(R_{T H}(t)\right)$ individuals. So that,

$$
N(t)=S+L_{H}+H_{U}+H_{D}+H_{W}+L_{T H}+A_{T H}+L_{H T}+A_{H T}+L_{T}+T_{U}+T_{D}+F+R_{T}+R_{T H}
$$

The Susceptible population is increased by the recruitment of individuals into the population at rate $\pi$.the population decrease by natural death rate $\mu$ and by both singly and dually-infected transmission individuals. Transmission by singly-infected individuals, we assumed that susceptible individuals acquire HIV infection, following effective contact with people infected with HIV only (i.e., those in the $\left(L_{H}, \eta_{U} H_{U}, \eta_{d H} H_{D}\right.$ and $\left.\eta_{W} H_{W}\right)$ classes at a rate $\lambda_{H}$, given by

$$
\lambda_{H}=\beta_{H} \frac{\left(L_{H}+\eta_{U} H_{U}+\eta_{d H} H_{D}+\eta_{W} H_{W}\right)}{N}
$$

Where, $\beta_{H}$ is the effective contact rate for HIV transmission.

Similarly, susceptible individuals acquire TB infection from individuals with TB only. $\left(L_{T}, \eta_{U} T_{U}, \eta_{d T} T_{D}, \eta_{R T} R_{T}\right.$ andF $\left.F_{T}\right)$ classes at a rate $\lambda_{T}$, given by

$\lambda_{T}=\beta_{T} \frac{\left(L_{T}+\eta_{U} T_{U}+\eta_{d T} T_{D}+\eta_{R T} R_{T}+F_{T}\right)}{N}$

Where, $\beta_{T}$ is the effective contact rate for TB infection.

Dually-infected individuals are assumed capable of transmitting either HIV or TB, but not the mixed infection.

$\lambda_{H T}=\beta_{H} \frac{\left(L_{H T}+\eta_{H} A_{H T}\right)}{N}$

$\lambda_{T H}=\beta_{T} \frac{\left(L_{T H}+\eta_{T} A_{T H}+\eta_{R T} R_{T H}\right)}{N}$

Then,

$\frac{d S}{d t}=\pi-\lambda_{H} S-\lambda_{T} S-\mu S-\lambda_{T H} S-\lambda_{H T} S$

A fraction $\varepsilon_{1}$ of the newly infected individuals with HIV only are assumed to show no disease symptoms initially. These individuals (known as "slow progressor" for HIV) are moved to the latently HIV class $\left(L_{H}\right)$. The remaining fraction, $\left(1-\varepsilon_{1}\right)$ of the newly infected individuals are assumed to immediately display disease symptoms (Fast 
progressors) and are moved to the undetected infectious class $H_{U}$. The population of latent class is decreased by the progression of latent HIV individual to active undetected HIV $H_{U}$ (at a rate $\kappa_{H}$ ) and also reduced by natural death rate $(\mu)$ and finally increased by the fraction of Treated HIV at the rate $(\phi)$ that moves from treated class to latently HIV compartment. Thus,

$$
\frac{d L_{H}}{d t}=\varepsilon_{1} \lambda_{H} S-\left(\kappa_{H}+\mu\right) L_{H}+\phi H_{W}
$$

The population of undetected infected individuals is increased by the infection of fast progressors (at the rate $\left.\left(1-\varepsilon_{1}\right) \lambda\right)$ and the development of symptoms by latently individual at the rate $\left(1-\omega_{1}\right) \kappa_{H}$ where $\omega_{1}$ is the endogenous reactivation rate. This population is decreased by natural death rate $(\mu)$ and disease induced death (at a rate $\left.\delta_{U H}\right)$ and further decreased by detection rate $\left(\gamma_{U H}\right)$ of HIV undetected infected individuals. Hence

$\frac{d H_{U}}{d t}=\left(1-\varepsilon_{1}\right) \lambda_{H} S+\left(1-\omega_{1}\right) \kappa_{H} L_{H}-\left(\gamma_{U H}+\mu+\delta_{U H}\right) H_{U}$

The population of Detected infected HIV individual increases by the fraction of latently individuals who develop disease symptoms (at the rate $\omega_{1} \kappa_{H}$ ), where $\omega_{1}$ is the endogenous reactivation rate and the detection of undetected individual at the rate $\gamma_{U H}$. The population later decreased by treatment rate $\left(\tau_{1}\right)$ for HIV detected individual and finally reduced by the natural death rate, induced mortality death rate at $\mu$ and $\delta_{U H}$ respectively. Hence we have.

$\frac{d H_{D}}{d t}=\omega_{1} \kappa_{H} L_{H}+\gamma_{U H} H_{U}-\left(\tau_{1}+\mu+\delta_{U H}\right) H_{D}$

The population of Treated HIV individuals is increased by those that have received treatment from HIV detected infected individual at the rate $\left(\tau_{1}\right)$ this population reduces by fraction of treated individual that moved back to latently HIV individuals at the rate, $(\phi)$ since treatment does not completely clears the bacteria and finally reduced by natural death rate $(\mu)$.

Hence,

$\frac{d H_{W}}{d t}=\tau_{1} H_{D}-(\phi+\mu) H_{W}$

The population of latent TB induced HIV is increased by infection, which can be acquired following effective contact with infections individuals in the latent TB induced HIV $\left(L_{T H}\right)$, Active TB induced HIV $\left(\eta_{T} A_{T H}\right)$ or Recovered TB induced HIV $\left(\eta_{R T} R_{T H}\right)$ categories at a rate $\lambda$ given by

$\lambda_{T H}=\beta_{T} \frac{\left(L_{T H}+\eta_{T} A_{T H}+\eta_{R T} R_{T H}\right)}{N}$

Where $\beta_{T}$ represents the effective contact rate. The population reduced by progression from latent stage to active stage at the rate $\left(\kappa_{T H}\right)$, and by natural death at the rate $(\mu)$. The population later increased by fraction of those that have been treated that moved from treated compartment at the rate $\left(\alpha_{T}\right)$. Then the rate of change of latent TB induced HIV population is given by;

$\frac{d L_{T H}}{d t}=\lambda_{T H} S-\left(\kappa_{T H}+\mu\right) L_{T H}+\alpha_{T} R_{T H}$

The population of active TB induced HIV is increased by the progression from latent stage to active stage at the rate $\left(\kappa_{T H}\right)$, the population decreased by natural death, induced mortality due to disease at the rate $(\mu)$ and $(\delta)$ respectively, individual who recovered also moved to recovered TB induced HIV at the rate $\left(\sigma_{1}\right)$.

Hence, 


$$
\frac{d A_{T H}}{d t}=\kappa_{T H} L_{T H}-\left(\mu+\sigma_{1}+\delta A_{T H}\right) A_{T H}
$$

The population of latent HIV induced TB is increased by infection, which can be acquired following effective contact with infectious individuals in the latent HIV induced TB $\left(L_{H T}\right)$, or active HIV induced TB $\left(\eta_{H} A_{H T}\right)$ categories at a rate $\lambda$ given by

$$
\lambda_{H T}=\beta_{H} \frac{\left(L_{H T}+\eta_{H} A_{H T}\right)}{N}
$$

Where $\beta_{H}$ represents the effective contact rate. The population reduced by progression from latent stage to active stage at the rate $\left(\kappa_{H T}\right)$ and by natural death rate. Hence, latent HIV induced TB population is given by

$$
\frac{d L_{H T}}{d t}=\lambda_{H T} S-\left(\kappa_{H T}+\mu\right) L_{H T}
$$

Active HIV induced TB (АНT) population is increased by the progression from latent stage to active stage at the rate $\left(\kappa_{H T}\right)$. The population decreased by natural death rate $(\mu)$ and disease induced mortality at the rate $(\delta)$. Hence the system of equation of Active HIV induced TB is given by

$$
\frac{d A_{H T}}{d t}=\kappa_{H T}-\left(\mu+\delta A_{H T}\right) A_{H T}
$$

A fraction $\varepsilon_{2}$ of the newly-infected individuals with TB only are assumed to show no disease symptoms initially. These individuals (Known as slow progressor for TB) moved to the latently TB class $\left(L_{T}\right)$. The population decreased by progression rate $\left(\kappa_{T}\right)$ from latent TB class to infected undetected class, natural death rate $(\mu)$ and exogenous re-infective (at a rate $\varphi \lambda_{T}$ ), where $\varphi<1$ accounts for the assumption that latent individual have reduced infection rate, this is to account for the fact that individuals with latent TB infection have partial immunity against exogenous re-infection. [29].The population increased by natural recovery at the rate $(v)$, number of unsuccessful treated individuals who move to the latent TB individual at the rate $\left(\theta_{1} \rho\right)$ and rate $(r \alpha)$ at which treated TB individual wanes off the treatment. Then the population is given by

$$
\frac{d L_{T}}{d t}=\varepsilon_{2} \lambda_{T} S-\left(\kappa_{T}+\varphi \lambda_{T}+\mu\right) L_{T}+v T_{U}+\theta_{1} \rho F_{T}+r \alpha R_{T}
$$

The population of undetected infectious individuals is increased by the infection of fast progressor at the rate $\left(1-\varepsilon_{1}\right) \lambda_{T}$ and the development of symptoms by latent individual at the rate $\left(1-\omega_{2}\right) \kappa_{T}$, where $\omega_{2}$ is the fraction of exposed individuals who develop symptoms and are detected. It is further increased by the exogenous re-infection of expressed individual at the rate $\left(1-\omega_{3}\right) \varphi \lambda_{T}$, where $\omega_{3}$ the fraction of is re-infected exposed individuals who are detected, and fraction of unsuccessful treated individuals that move from detected individuals to undetected individuals at the rate $\left(\theta_{2} \rho\right)$. This population is decreased by natural recovery at a rate $(\mathrm{v})$, detection of undetected individual at a rate $\left(\gamma_{U H}\right)$, natural death at the rate $(\mu)$ and disease induced death at a rate $\left(\delta_{U T}\right)$.

Hence,

$$
\frac{d T_{U}}{d t}=\left(1-\varepsilon_{2}\right) \lambda_{H} S+\left(1-\omega_{2}\right) \kappa_{T} L_{T}+\left(1+\omega_{3}\right) \varphi \lambda_{T} L_{T}+\theta_{2} \rho F_{T}-\left(v+\gamma_{U T}+\mu+\delta_{U T}\right) T_{U}
$$

The population of detected infectious individual increases by the fraction of latent individuals who develop diseases symptoms at a rate $\omega_{2} \kappa_{T}$, exogenous re-infection of latent individual, detection rate for undetected individual at the rate $\left(\omega_{3} \varphi \lambda_{T}, \gamma_{U T}\right.$ respectively) and numbers of unsuccessful treated individuals who move to latent and undetected individual at the rate $\left(\theta_{1}\right)$ and $\left(\theta_{2}\right)$ respectively. The population is decreased by those that are treated and recovered 
who later moved to recovered compartment at the rate $\left(\sigma_{2}\right)$, treatment rate $\left(\tau_{1}\right)$, natural death rate $(\mu)$ and disease induced death at a rate $\left(\delta_{d T}\right)$. This gives

$$
\frac{d T_{D}}{d t}=\omega_{2} \kappa_{T} L_{T}+\omega_{3} \varphi \lambda_{T} L_{T}+\gamma_{U T} T_{U}+\left[1-\left(\theta_{1}+\theta_{2}\right)\right] \rho F_{T}-\left(\sigma_{2}+\tau_{2}+\mu+\delta_{d T}\right) T_{D}
$$

The population of fail treatment compartment is generated by the failure of treated infected detected individuals at the rate $\left(1-q_{1}\right) \tau_{2}$. The treatment failure could be due to a number of reasons, such as incomplete compliance to the specified treatment or drug resistance among others. The population decreased by fraction of treated individuals who lose their treatment the population decreased by the rate at which TB individuals who fail treatment move to other classes, the natural death and induced death at the rate $\left(\rho+\mu+\delta_{F}\right)$. Thus,

$$
\frac{d F_{T}}{d t}=\left(1-q_{1}\right) \tau_{2} T_{D}+(1-r) \alpha R_{T}-\left(\rho+\mu+\delta_{F}\right) F_{T}
$$

The population of TB recovered individual is increased by the treatment of detected individual at the rate $q_{1} \tau_{2}$ and treated detected individual (at the rate $\sigma_{2}$ ), successfully- recovered individuals eventually move to the latent class (at the rate $\alpha$ ). This population is further decreased by natural death and disease induced death (at a rate $\mu$ and $\delta_{R T}$ ).

Hence

$$
\frac{d R_{T}}{d t}=q_{1} \tau_{2} T_{D}-\left(\alpha+\mu+\delta_{R T}\right) R_{T}+\sigma_{2} T_{D}
$$

The population of TB induced HIV recovered individual is increased by the treatment of active TB at the $\left(\sigma_{1}\right)$ and leather reduced by natural death rate at $\mu$ and rate $\left(\alpha_{T}\right)$, at which treated individual lose their treatment induced immunity. Then we have

$$
\frac{d R_{T H}}{d t}=\sigma_{1} A_{T H}-\left(\alpha_{T}+\mu\right) R_{T H}
$$

Hence, we have, 


\subsection{MATHEMATICAL MODEL OF HIV-TB}

$$
\begin{aligned}
& \frac{d S}{d t}=\pi-\lambda_{H} S-\lambda_{T} S-\mu S-\lambda_{T H} S-\lambda_{H T} S \\
& \frac{d L_{H}}{d t}=\varepsilon_{1} \lambda_{H} S-K_{1} L_{H}+\phi H_{W} \\
& \frac{d H_{U}}{d t}=\left(1-\varepsilon_{1}\right) \lambda_{H} S+K_{2} L_{H}-K_{3} H_{U} \\
& \frac{d H_{D}}{d t}=\omega_{1} \kappa_{H} L_{H}+\gamma_{U H} H_{U}-K_{4} H_{D} \\
& \frac{d H_{W}}{d t}=\tau_{1} H_{D}-K_{5} H_{W} \\
& \frac{d L_{T H}}{d t}=\lambda_{T H} S-K_{6} L_{T H}+\alpha_{T} R_{T H} \\
& \frac{d A_{T H}}{d t}=\kappa_{T H} L_{T H}-K_{7} A_{T H} \\
& \frac{d L_{H T}}{d t}=\lambda_{H T} S-K_{8} L_{H T} \\
& \frac{d A_{H T}}{d t}=\kappa_{H T} L_{H T}-K_{9} A_{H T} \\
& \frac{d L_{T}}{d t}=\varepsilon_{2} \lambda_{T} S-K_{10} L_{T}+v T_{U}+\theta_{1} \rho F_{T}+r \alpha R_{T} \\
& \frac{d T_{U}}{d t}=\left(1-\varepsilon_{2}\right) \lambda_{H} S+K_{11} L_{T}+K_{12} L_{T}+\theta_{2} \rho F_{T}-K_{13} T_{U} \\
& \frac{d T_{D}}{d t}=\omega_{2} \kappa_{T} L_{T}+\omega_{3} \varphi \lambda_{T} L_{T}+\gamma_{U T} T_{U}+K_{14} F_{T}-K_{15} T_{D} \\
& \frac{d F_{T}}{d t}=K_{16} T_{D}+K_{17} R_{T}-K_{18} F_{T} \\
& \frac{d R_{T}}{d t}=K_{19} T_{D}-K_{20} R_{T} \\
& =\sigma_{1} A_{T H}-K_{21} R_{T H} \\
& \frac{d t}{d}{ }_{1}
\end{aligned}
$$

Where

$K_{1}=\left(\kappa_{H}+\mu\right), \quad K_{2}=\left(1-\omega_{1}\right) \kappa_{H}, \quad K_{3}=\left(\gamma_{U H}+\mu+\delta_{U H}\right), \quad K_{4}=\left(\tau_{1}+\mu+\delta_{d H}\right), K_{5}=(\phi+\mu)$,

$K_{6}=\left(\kappa_{T H}+\mu\right), \quad K_{7}=\left(\mu+\sigma_{1}+\delta A_{T H}\right), \quad K_{8}=\left(\kappa_{H T}+\mu\right), \quad K_{9}=\left(\mu+\delta A_{H T}\right), K_{10}=\left(\kappa_{T}+\varphi \lambda_{T}+\mu\right)$,

$K_{11}=\left(1-\omega_{2}\right) \kappa_{T}, K_{12}=\left(1+\omega_{3}\right) \varphi \lambda_{T}, K_{13}=\left(v+\gamma_{U T}+\mu+\delta_{U T}\right), \quad K_{14}=\left[1-\left(\theta_{1}+\theta_{2}\right)\right] \rho$,

$K_{15}=\left(\sigma_{2}+\tau_{2}+\mu+\delta_{d T}\right), K_{16}=\left(1-q_{1}\right) \tau_{2}, \quad K_{17}=(1-r) \alpha, K_{18}=\left(\rho+\mu+\delta_{F}\right), K_{19}=\left(q_{1} \tau_{2}+\sigma_{2}\right)$,

$K_{20}=\left(\alpha+\mu+\delta_{R T}\right), \quad K_{21}=\left(\alpha_{T}+\mu\right)$

\begin{tabular}{cl} 
VARIABLES AND PARAMETERS DESCRIPTIONS \\
VARIABLES & \multicolumn{1}{c}{ DESCRIPTIONS } \\
$\mathrm{S}$ & Susceptible individuals \\
$L_{H}$ & Latent HIV individual \\
$H_{D}$ & Detected HIV individual \\
$H_{U}$ & Undetected HIV individual
\end{tabular}




$L_{T H}$
$A_{T H}$
$L_{T}$
$T_{U}$
$T_{D}$
$F_{T}$
$R_{T}$
$R_{T H}$
$H_{W}$
$L_{H T}$
$A_{H T}$

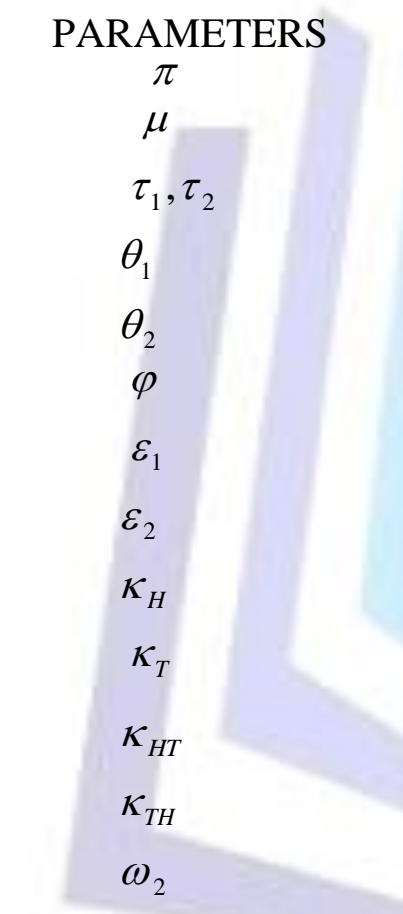

$\omega_{3}$
$\gamma_{U H}$
$\gamma_{U T}$
$v$
$\alpha$
$\mathrm{r}$
$\rho$
$\delta_{U T}, \delta_{d T}, \delta_{F}, \delta_{R T}$
$\phi$
$\sigma_{2}$
$\sigma_{1}$

Latent TB induced HIV individual

Active TB induced HIV individual

Latent TB individual

Undetected TB individual

Detected TB individual

TB treatment fail individual

TB recover individual

TB induced HIV recover individual

Treated HIV individual

Latent HIV induced TB

Active HIV induced TB

\section{DESCRIPTIONS}

Recruitment rate

Natural death rate

Treatment rate for Detected HIV and TB

Number of unsuccessful treated individual who moves to latent TB

Number of unsuccessful treated who moves to undetected TB

Exogenous re-infection rate for TB

Fast progressor for HIV

Fast progressors for TB

Progression rate of HIV.

Progression rate of TB.

Progression rate of HIV induced TB

Progression rate of TB induced HIV

Endogenous reactivation rate of TB

Fraction of re-infected individuals that moves to detected class

Detection rate for undetected HIV

Detection rate for undetected TB

Natural recovery rate of undetected TB individuals

Loss of immunity

Treated TB who move to Latent class after treatment wanes

Rate at which TB individuals fail treatment.

Tuberculosis-induced mortality rate for classes $T_{U}, T_{D}, F, R$

HIV treated individuals that moves to Latent individuals

Recovery rate for TB detected individuals

Recovery rate for active TB induced HIV individuals 

$\beta_{T}, \beta_{H}$
Effective contact rate for TB and HIV
$q_{1}$
$\delta_{U H}, \delta_{d H}$
$\eta_{U}, \eta_{d H}, \eta_{W}$
$\eta_{U}, \eta_{d T}, \eta_{F T}, \eta_{R T}$
Detected individuals who are successfully treated HIV-induced mortality rate for classes $H_{U}, H_{D}$ respectively HIV Modification parameters for classes $H_{U}, H_{D}, H_{W}$ respectively
TB Modification parameters for classes $T_{U}, T_{D}, F_{T}, R_{T}$ respectively

\subsection{POSITIVITY OF SOLUTIONS}

For HIV-TB model only to be epidemiologically meaningful and well posed, we need to prove that all state variables are non-negative for all $t>0$

$D_{1}=\left\{\left(S, L_{H}, H_{U}, H_{D}, H_{W}, L_{T H}, A_{T H}, L_{H T}, A_{H T}, L_{T}, T_{U}, T_{D}, F_{T}, R_{T}, R_{T H}\right) \in R_{+}^{15}: N \leq \pi / \mu\right\}$

Lemma 1.

The closed set

$D_{1}=\left\{\left(S, L_{H}, H_{U}, H_{D}, H_{W}, L_{T H}, A_{T H}, L_{H T}, A_{H T}, L_{T}, T_{U}, T_{D}, F_{T}, R_{T}, R_{T H}\right) \in R_{+}^{15}: N \leq \pi / \mu\right\}$ is positively-invariant and attracting with respect to the model (23)

Proof: Consider the biologically-feasible region $D$, defined above. The rate of change of the total population, obtained by adding all equations of the model (23), is given by

$\frac{d N}{d t}=\pi-\mu N-\delta_{U H}-\delta_{d H}-\delta A_{T H}-\delta A_{H T}-\delta_{U T}-\delta_{d T}-\delta_{R T}-\delta_{F}$.

It follows that $\frac{d N}{d t}<0$ whenever $N>\frac{\pi}{\mu}$. Furthermore, since $\frac{d N}{d t} \leq \pi-\mu N$, it is clear that $N(t) \leq \frac{\pi}{\mu}$ if

$N(0) \leq \frac{\pi}{\mu}$. Therefore, all solutions of the model with initial conditions in $D$ remain in $D$ for all $t>0$ (i.e., the $\omega$-limits sets of the system (13) are contained in $D$ ). Thus, $D$ is positively-invariant and attracting. In this region, the model can be considered as been epidemiologically and mathematically well posed

\subsection{ANALYSIS OF SUB MODEL}

Before analyzing the full model (23), it is pertinent to gain insights into the dynamics of the models for HIV only and TB only.

\subsection{HIV MODEL ONLY}

$\frac{d S}{d t}=\pi-\lambda_{H} S-\mu S$

$\frac{d L_{H}}{d t}=\varepsilon_{1} \lambda_{H} S-K_{1} L_{H}+\phi H_{W}$

$\frac{d H_{U}}{d t}=\left(1-\varepsilon_{1}\right) \lambda_{H} S+K_{2} L_{H}-K_{3} H_{U}$

$\frac{d H_{D}}{d t}=\omega_{1} \kappa_{H} L_{H}+\gamma_{U H} H_{U}-K_{4} H_{D}$

$\frac{d H_{W}}{d t}=\tau_{1} H_{D}-K_{5} H_{W}$

Where $\lambda_{H}=\beta_{H} \frac{\left(L_{H}+\eta_{U} H_{U}+\eta_{d H} H_{D}+\eta_{W} H_{W}\right)}{N}$ 
For this model, it can be shown that the region,

$D_{1}=\left\{\left(S+L_{H}+\eta_{U} H_{U}+\eta_{d H} H_{D}+\eta_{W} H_{W}\right) \in R_{+}^{5}: N \leq \pi / \mu\right.$

\subsubsection{DERIVATION OF BASIC REPRODUCTION NUMBER $\left(\mathbf{R}_{0}\right)$ FOR HIV ONLY The Next Generation Matrix (F. $\left.\mathbf{V}^{-1}\right)$ Method}

One of fundamental questions of mathematical epidemiology is to find the threshold conditions that determine whether an infectious disease will spread in a population when the disease is introduced into the population [14].

The basic reproduction number of the model (25) $R_{H}$ is calculated by using the next generation matrix [31]. Using their approach [31], we have,

$$
F_{i}=\left(\begin{array}{l}
F 1 \\
F 2 \\
F 3 \\
F 4
\end{array}\right)=\left(\begin{array}{l}
\varepsilon_{1} \lambda_{H} S \\
\left(1-\varepsilon_{1}\right) \lambda_{H} S \\
0 \\
0
\end{array}\right) \text { and } V_{i}=\left(\begin{array}{l}
V 1 \\
V 2 \\
V 3 \\
V 4
\end{array}\right)=\left(\begin{array}{l}
\left(K_{H}+\mu\right) L_{H}-\phi H_{W} \\
\left(1-\omega_{1}\right) K_{H} L_{H}+\left(\gamma_{U H}+\mu+\delta_{U H}\right) H_{U} \\
-\omega_{1} K_{H} L_{H}-\gamma_{U H} H_{U}+\left(\tau_{1}+\mu+\delta_{U H}\right) H_{D} \\
-\tau_{1} H_{D}+(\phi+\mu) H_{W}
\end{array}\right)
$$

After taking partial derivative $\mathrm{F}$ and $\mathrm{V}$, we have

$$
\begin{aligned}
& \mathrm{F}=\left(\begin{array}{llcl}
\varepsilon_{1} \beta_{H} & \varepsilon_{1} \beta_{H} \eta_{U} & \varepsilon_{1} \beta_{H} \eta_{d H} & \varepsilon_{1} \beta_{H} \eta_{W} \\
\left(1-\varepsilon_{1}\right) \beta_{H} & \left(1-\varepsilon_{1}\right) \beta_{H} \eta_{U} & \left(1-\varepsilon_{1}\right) \beta_{H} \eta_{d H} & \left(1-\varepsilon_{1}\right) \beta_{H} \eta_{W} \\
0 & 0 & 0 & 0 \\
0 & 0 & 0 & 0
\end{array}\right) \\
& \mathrm{V}=\left(\begin{array}{llll}
K_{1} & 0 & 0 & -\phi \\
K_{2} & K_{3} & 0 & 0 \\
-\omega_{1} K_{H} & -\gamma_{U H} & K_{4} & 0 \\
0 & 0 & -\tau_{1} & K_{5}
\end{array}\right)
\end{aligned}
$$

Thus,

$$
R_{H}=\left\{\left(\frac{1}{\phi \tau_{1} K_{2} \gamma_{U H}+\phi \tau_{1} \omega_{1} K_{H} K_{3}-K_{4} K_{5} K_{1} K_{3}}\right)\left(\begin{array}{l}
\beta_{H}\left(-\left(1-\varepsilon_{1}\right) \gamma_{U H} \phi \tau_{1}+\left(1-\varepsilon_{1}\right) \eta_{U} \phi \tau_{1} \omega_{1} \kappa_{H}\right. \\
-\left(1-\varepsilon_{1}\right) \eta_{U} K_{5} K_{4} K_{1}-\left(1-\varepsilon_{1}\right) \gamma_{U H} \eta_{d H} K_{5} K_{1} \\
-\left(1-\varepsilon_{1}\right) \gamma_{U H} \eta_{W} \tau_{1} K_{1}-\varepsilon_{1} K_{4} K_{5} K_{3}-\varepsilon_{1} K_{4} K_{5} K_{2} \eta_{U} \\
-\eta_{d H} \omega_{1} \kappa_{H} \varepsilon_{1} K_{5} K_{3}-\eta_{d H} \gamma_{U H} \varepsilon_{1} K_{5} K_{2}-\tau_{1} \omega_{1} \kappa_{H} \varepsilon_{1} \eta_{W} K_{3} \\
-\eta_{W} \gamma_{U H} \varepsilon_{1} \tau_{1} K_{2}
\end{array}\right)\right\}
$$

The threshold quantity $R_{H}$ is the basic reproduction number of the normalized model system (25) for HIV infection in a population. It measures the average number of new secondary infections generated by a single infected individual in his or her infectious period in a susceptible population [1].

\subsubsection{DISEASE FREE EQUILIBRIUM}

For critical points, we set

$\frac{d S}{d t}=\frac{d L_{H}}{d t}=\frac{d H_{U}}{d t}=\frac{d H_{D}}{d t}=\frac{d H_{W}}{d t}=0$

At disease free equilibrium, it is assumed that there is no infection, Hence (DFE) is given as

$\varepsilon_{0}=\left(S, L_{H}, H_{U}, H_{D}, H_{W}\right)=\left(\frac{\pi}{\mu}, 0,0,0,0\right)$ 


\subsubsection{GLOBAL STABILITY OF DISEASE FREE EQUILIBRIUM (HIV)}

Here, the global asymptotic stability (GAS) property of the DEF of the HIV model only will be explored.

Theorem3.4: The disease free-equilibrium of the system (25) is globally asymptotically stable whenever $R_{H}<1$ and unstable if $R_{H}>1$.

Proof: It follows that $S=N^{*}-L_{H}-H_{U}-H_{D}-H_{W}$ at steady state. The proof is based on using the comparison method [21] to prove the global stability.

Using comparison method, we have,

$\left(\begin{array}{l}\frac{d L_{H}}{d t} \\ \frac{d H_{U}}{d t} \\ \frac{d H_{D}}{d t} \\ \frac{d H_{W}}{d t}\end{array}\right)=(F-V)\left(\begin{array}{l}L_{H} \\ H_{U} \\ H_{D} \\ H_{W}\end{array}\right)-F i\left(\begin{array}{l}L_{H} \\ H_{U} \\ H_{D} \\ H_{W}\end{array}\right)$

Where

$F-V=\left(\begin{array}{cccc}\varepsilon_{1} \beta_{H}-K_{1} & \varepsilon_{1} \beta_{H} \eta_{U} & \varepsilon_{1} \beta_{H} \eta_{d H} & \varepsilon_{1} \beta_{H} \eta_{W}+\phi \\ \left(1-\varepsilon_{1}\right) \beta_{H}+K_{2} & \left(1-\varepsilon_{1}\right) \beta_{H} \eta_{U}-K_{3} & \left(1-\varepsilon_{1}\right) \beta_{H} \eta_{d H} & \left(1-\varepsilon_{1}\right) \beta_{H} \eta_{W} \\ \omega_{1} \kappa_{H} & \gamma_{U H} & -K_{4} & 0 \\ 0 & 0 & \tau_{1} & -K_{5}\end{array}\right)$

According to $[6,31]$, all eigen values of the matrix $(F-V)$ have negative real parts.

Hence, we have

$\left[\begin{array}{l}\left(\left(\varepsilon_{1} \beta_{H}-K_{1}\right)-\lambda\right)\left(\left(1-\varepsilon_{1}\right) \beta_{H} \eta_{U}-K_{3}-\lambda\right)\left(-K_{4}-\lambda\right)\left(-K_{5}-\lambda\right) \\ -\left(\varepsilon_{1} \beta_{H} \eta_{U}\right)\left(\left(1-\varepsilon_{1}\right) \beta_{H}+K_{2}\right)\left(-K_{4}-\lambda\right)\left(-K_{5}-\lambda\right)+\left(\varepsilon_{1} \beta_{H} \eta_{d H}\right)\left(\left(1-\varepsilon_{1}\right) \beta_{H}+K_{2}\right)\left(\gamma_{U H}\left(-K_{5}-\lambda\right)\right) \\ -\left(\varepsilon_{1} \beta_{H} \eta_{W}+\phi\right)\left(\left(1-\varepsilon_{1}\right) \beta_{H}+K_{2}\right)\left(\gamma_{U H}+\tau_{1}\right)\end{array}\right]=0$

Thus, from equation (32), the characteristic equation is given by

$\lambda^{4}+a_{4} \lambda^{3}+a_{3} \lambda^{2}+a_{2} \lambda-a_{1}$

Where

$$
\begin{aligned}
a_{4}= & \left(-\eta_{U} \beta_{H}\left(1-\varepsilon_{1}\right)-\varepsilon_{1} \beta_{H}+K_{4} K_{5} K_{1} K_{3}\right) \\
a_{3} & =\left(-\left(1-\varepsilon_{1}\right) K_{5} \eta_{U} \beta_{H}-K_{5} \varepsilon_{1} \beta_{H}+K_{5} K_{1}+K_{5} K_{3}+K_{4} K_{5}-\eta_{d H} \omega_{1} \kappa_{H} \varepsilon_{1} \beta_{H}-\left(1-\varepsilon_{1}\right) \gamma_{U H} \eta_{d H} \beta_{H}\right. \\
& \left.-\left(1-\varepsilon_{1}\right) K_{4} \eta_{U} \beta_{H}-K_{4} \varepsilon_{1} \beta_{H}+K_{4} K_{1}+K_{4} K_{3}-\left(1-\varepsilon_{1}\right) K_{1} \eta_{U} \beta_{H}-\varepsilon_{1} K_{2} \eta_{U} \beta_{H}-\varepsilon_{1} K_{3} \beta_{H}+K_{1} K_{3}\right) \\
a_{2}= & \left(-\tau_{1} \omega_{1} \kappa_{H} \varepsilon_{1} \eta_{W} \beta_{H}-\left(1-\varepsilon_{1}\right) \gamma_{U H} \eta_{W} \tau_{1} \beta_{H}-\phi \tau_{1} \omega_{1} \kappa_{H}-\eta_{d H} \omega_{1} \kappa_{H} \varepsilon_{1} K_{5} \beta_{H}-\left(1-\varepsilon_{1}\right) \gamma_{U H} \eta_{d H} K_{5} \beta_{H}\right. \\
& -\left(1-\varepsilon_{1}\right) \eta_{U} K_{5} K_{4} \beta_{H}-K_{4} K_{5} \varepsilon_{1} \beta_{H}+K_{4} K_{5} K_{1}+K_{4} K_{5} K_{3}-\left(1-\varepsilon_{1}\right) \eta_{U} \beta_{H} K_{5} K_{1}-\varepsilon_{1} K_{2} K_{5} \eta_{U} \beta_{H} \\
& -\varepsilon_{1} K_{3} K_{5} \beta_{H}+-K_{3} K_{2} K_{5}-\eta_{d H} \gamma_{U H} \varepsilon_{1} K_{2} \beta_{H}-\left(1-\varepsilon_{1}\right) K_{1} \gamma_{U H} \eta_{d H} \beta_{H}-\eta_{d H} \kappa_{H} \varepsilon_{1} K_{3} \beta_{H} \omega_{1} \\
& \left.-\left(1-\varepsilon_{1}\right) \eta_{U} \beta_{H} K_{4} K_{1}-\eta_{U} \varepsilon_{1} K_{2} K_{4} \beta_{H}-\varepsilon_{1} K_{3} K_{4} \beta_{H}+K_{4} K_{1} K_{3}\right) \\
a_{1}= & \left(-\left(-\phi \tau_{1} \omega_{1} \kappa_{H} K_{3}-\varepsilon_{1} K_{4} K_{5} K_{3} \beta_{H}-\phi \tau_{1} K_{2} \gamma_{U H}+K_{4} K_{5} K_{1} K_{3}-\left(1-\varepsilon_{1}\right) \gamma_{U H} \eta_{W} \tau_{1} K_{1} \beta_{H}\right.\right. \\
& -\left(1-\varepsilon_{1}\right) \gamma_{U H} \eta_{d H} K_{5} K_{1} \beta_{H}-\left(1-\varepsilon_{1}\right) \eta_{U} K_{5} K_{4} K_{1} \beta_{H}+\left(1-\varepsilon_{1}\right) \eta_{U} \phi \tau_{1} \omega_{1} \kappa_{H} \beta_{H}-\left(1-\varepsilon_{1}\right) \gamma_{U H} \phi \tau_{1} \beta_{H} \\
& \left.\left.-\eta_{d H} \gamma_{U H} \varepsilon_{1} K_{5} K_{2} \beta_{H}-\varepsilon_{1} K_{4} K_{5} K_{2} \eta_{U} \beta_{H}-\eta_{W} \gamma_{U H} \varepsilon_{1} \tau_{1} K_{2} \beta_{H}-\tau_{1} \omega_{1} \kappa_{H} \varepsilon_{1} \eta_{W} K_{3} \beta_{H}-\eta_{d H} \omega_{1} \kappa_{H} \varepsilon_{1} K_{5} K_{3}\right)\right)
\end{aligned}
$$

Applying Routh-Hurwitz criteria of order 4; 
$n=4: a_{1}>0, a_{3}>0, a_{4}>0$, and $a_{1} a_{2} a_{3}>a_{3}^{2}+a_{1}^{2} a_{4}$.

Then for $a_{1}>0$ we have

$$
-\left(\begin{array}{l}
\beta_{H}\left(-\left(1-\varepsilon_{1}\right) \gamma_{U H} \phi \tau_{1}+\left(1-\varepsilon_{1}\right) \eta_{U} \phi \tau_{1} \omega_{1} \kappa_{H}\right. \\
-\left(1-\varepsilon_{1}\right) \eta_{U} K_{5} K_{4} K_{1}-\left(1-\varepsilon_{1}\right) \gamma_{U H} \eta_{d H} K_{5} K_{1} \\
-\left(1-\varepsilon_{1}\right) \gamma_{U H} \eta_{W} \tau_{1} K_{1}-\varepsilon_{1} K_{4} K_{5} K_{3}-\varepsilon_{1} K_{4} K_{5} K_{2} \eta_{U} \\
-\eta_{d H} \omega_{1} \kappa_{H} \varepsilon_{1} K_{5} K_{3}-\eta_{d H} \gamma_{U H} \varepsilon_{1} K_{5} K_{2}-\tau_{1} \omega_{1} \kappa_{H} \varepsilon_{1} \eta_{W} K_{3} \\
\left.-\eta_{W} \gamma_{U H} \varepsilon_{1} \tau_{1} K_{2}\right)
\end{array}\right)+\left(\phi \tau_{1} K_{2} \gamma_{U H}+\phi \tau_{1} \omega_{1} K_{H} K_{3}-K_{4} K_{5} K_{1} K_{3}\right)>0
$$

Hence

$\frac{\left(\begin{array}{l}\beta_{H}\left(-\left(1-\varepsilon_{1}\right) \gamma_{U H} \phi \tau_{1}+\left(1-\varepsilon_{1}\right) \eta_{U} \phi \tau_{1} \omega_{1} \kappa_{H}-\left(1-\varepsilon_{1}\right) \eta_{U} K_{5} K_{4} K_{1}\right. \\ -\left(1-\varepsilon_{1}\right) \gamma_{U H} \eta_{d H} K_{5} K_{1}-\left(1-\varepsilon_{1}\right) \gamma_{U H} \eta_{W} \tau_{1} K_{1}-\varepsilon_{1} K_{4} K_{5} K_{3}-\varepsilon_{1} K_{4} K_{5} K_{2} \eta_{U}- \\ \eta_{d H} \omega_{1} \kappa_{H} \varepsilon_{1} K_{5} K_{3}-\eta_{d H} \gamma_{U H} \varepsilon_{1} K_{5} K_{2}-\tau_{1} \omega_{1} \kappa_{H} \varepsilon_{1} \eta_{W} K_{3}-\eta_{W} \gamma_{U H} \varepsilon_{1} \tau_{1} K_{2}\end{array}\right)}{\left(\phi \tau_{1} K_{2} \gamma_{U H}+\phi \tau_{1} \omega_{1} K_{H} K_{3}-K_{4} K_{5} K_{1} K_{3}\right)}<1$,

Hence, we have established that the disease free equilibrium is globally asymptotically stable whenever $R_{H}<1$ and unstable $R_{H}>1$.

\subsubsection{EXISTENCE OF ENDEMIC EQUILIBRIUM (EE)}

Where $\varepsilon_{0}^{* *}=\left(S^{* * *}, L_{H}^{* *}, H_{U}^{* *}, H_{D}^{* *}, H_{W}^{* *}\right)$ are the endemic equilibrium points.

Equation (25) becomes

$\frac{d S}{d t}=\pi-\lambda_{H} S-\mu S$

$\frac{d L_{H}}{d t}=\varepsilon_{1} \lambda_{H} S-K_{1} L_{H}+\phi H_{W}$

$\frac{d H_{U}}{d t}=\left(1-\varepsilon_{1}\right) \lambda_{H} S+K_{2} \kappa_{H} L_{H}-K_{3} H_{U}$

$\frac{d H_{D}}{d t}=\omega_{1} \kappa_{H} L_{H}+\gamma_{U H} H_{U}-K_{4} H_{D}$

$\frac{d H_{W}}{d t}=\tau_{1} H_{D}-K_{5} H_{W}$

Solving at steady state, from equation $(33.1-33.5)$, we have;

$\pi-\left(\lambda_{H}-\mu\right) S=0$

$S^{* *}=\frac{\pi}{\left(\lambda_{H}+\mu\right)}$

$L_{H^{* *}}=\frac{\varepsilon_{1} \lambda_{H} S^{* *}+\phi H_{W^{* *}}}{K_{1}}$ 


$$
\begin{aligned}
& H_{U^{* * *}}=\frac{\left(1-\varepsilon_{1}\right) \lambda_{H} S^{* *}+K_{2} \kappa_{H} L_{H}}{K_{3}} \\
& H_{D^{* *}}=\frac{\omega_{1} \kappa_{H} L_{H}^{* *}+\gamma_{U H} H_{U^{* * *}}}{K_{4}} \\
& H_{W^{* *}}=\frac{\tau_{1} H_{D^{* * *}}}{K_{5}}
\end{aligned}
$$

Substitute (33.10) into (33.7), we have

$$
\begin{aligned}
& L_{H^{* *}}=\frac{\varepsilon_{1} \lambda_{H^{* *}} S^{* *}}{X}+\frac{\phi \tau_{1} \gamma_{U H}\left(1-\varepsilon_{1}\right) \lambda_{H}^{* * *} S^{* *}}{Y} \\
& L_{H^{* *}}=\rho_{1} \lambda_{H}^{* *} S^{* *} \\
& H_{U^{* *}}=\frac{\left(1-\varepsilon_{1}\right) \lambda_{H}^{* *} S^{* *}}{K_{3}}+\frac{K_{12} K_{H} P_{1} \lambda_{H}^{* *} S^{* *}}{K_{3}} .=P_{2} \lambda_{H}^{* *} S^{* *} \\
& H_{D^{* *}}=\frac{\omega_{1} K_{H} P_{1} \lambda_{H}^{* *} S^{* *}}{K_{4}}+\frac{\gamma_{U H} P_{2} \lambda_{H}^{* *} S^{* *}}{K_{4}} .=P_{3} \lambda_{H}^{* *} S^{* *} \\
& H_{W}=\frac{\tau_{1} P_{3} \lambda_{H}^{* *} S^{* *}}{K_{5}} .=P_{4} \lambda_{H}^{* *} S^{* *}
\end{aligned}
$$

Where

$P_{1}=\frac{\varepsilon_{1}}{X}+\frac{\phi \tau_{1} \gamma_{U H}\left(1-\varepsilon_{1}\right)}{Y}$

$P_{2}=\frac{\left(1-\varepsilon_{1}\right)}{K_{3}}+\frac{K_{2} \kappa_{H}}{K_{3}}\left[\frac{\varepsilon_{1}}{X}+\frac{\phi \tau_{1} \gamma_{U H}\left(1-\varepsilon_{1}\right)}{Y}\right]$

$P_{3}=\frac{\omega_{1} \kappa_{H}}{K_{4}}\left[\frac{\varepsilon_{1}}{X}+\frac{\phi \tau_{1} \gamma_{U H}\left(1-\varepsilon_{1}\right)}{Y}\right]+\frac{\gamma_{U H}}{K_{4}}\left[\frac{\left(1-\varepsilon_{1}\right)}{K_{2}}+\frac{K_{2} \kappa_{H}}{K_{3}}\left[\frac{\varepsilon_{1}}{X}+\frac{\phi \tau_{1} \gamma_{U H}\left(1-\varepsilon_{1}\right)}{Y}\right]\right]$

$P_{4}=\frac{\tau_{1}}{K_{5}} \cdot \frac{\omega_{1} \kappa_{H}}{K_{4}}\left[\frac{\varepsilon_{1}}{X}+\frac{\phi \tau_{1} \gamma_{U H}\left(1-\varepsilon_{1}\right)}{Y}\right]+\frac{\gamma_{U H}}{K_{4}}\left[\frac{\left(1-\varepsilon_{1}\right)}{K_{2}}+\frac{K_{12} \kappa_{H}}{K_{3}}\left[\frac{\varepsilon_{1}}{X}+\frac{\phi \tau_{1} \gamma_{U H}\left(1-\varepsilon_{1}\right)}{Y}\right]\right]$

\section{Where}

$$
X=K_{1}\left(1-\frac{\phi \tau_{1} w_{1} \kappa_{H}}{K_{1} K_{4} K_{5}}-\frac{\phi \tau_{1} \gamma_{U H} K_{12} \kappa_{H}}{K_{1} K_{4} K_{5} K_{3}}\right)
$$

$Y=K_{1} K_{4} K_{5} K_{3}\left(1-\frac{\phi \tau_{1} \omega_{1} K_{H}}{K_{1} K_{4} K_{5}}-\frac{\phi \tau_{1} \gamma_{U H} K_{12} K_{H}}{K_{1} K_{4} K_{5} K_{3}}\right)$

$\lambda_{H}^{* *}=\frac{\beta_{H}\left[L_{H}^{* *}+\eta_{U} H_{U}^{* *}+\eta_{d H} H_{D}^{* *}+\eta_{W} H_{W}^{* *}\right]}{N}$

Substituting the expressions in (33.14-33.17) into (33.18) we have

$\lambda_{H}^{* *}\left\lfloor S^{* *}+P_{1} \lambda_{H}^{* *} S^{* *}+P_{2} \lambda_{H}^{* * * * *} S^{* *} P_{3} \lambda_{H}^{* * * * * *} S^{* *} P_{4} \lambda_{H}^{* * * * *} S^{* *}\right]=\beta \lambda_{H}^{* *} S^{* *}\left[P_{1}+\eta_{U} P_{2}+\eta_{d H} P_{3}+\eta_{W} P_{4}\right]$

Divide each term in (33.19) by $\lambda_{H}{ }^{* *} S^{* *}$

$1+P_{5} \lambda^{* *}=\beta\left[P_{1}+\eta_{U} P_{2}+\eta_{d H} P_{3}+\eta_{W} P_{4}\right]$ 
Where $P_{5}=P_{1}+P_{2}+P_{3}+P_{4} \geq 0$

So that

$1+P_{5} \lambda_{H}^{* * *}=\frac{\beta}{K_{1} K_{3} K_{4} K_{5}}\left[\frac{\varepsilon_{1}\left(K_{3} K_{4} K_{5}\right)}{K_{1} A}+\frac{\phi \tau_{1} \gamma_{U H}\left(1-\varepsilon_{1}\right)}{(A)}+\left(1-\varepsilon_{1}\right) K_{1} K_{4} K_{5}\right.$

$+\frac{K_{2} K_{H} \varepsilon_{1} K_{4} K_{5}}{A}+\frac{\phi \tau_{1} \gamma_{U H}\left(1-\varepsilon_{1}\right)}{A}+\frac{\omega_{1} K_{H} \varepsilon_{1} K_{3} K_{5}}{A}+\frac{\phi \tau_{1} \gamma_{U H}\left(1-\varepsilon_{1}\right)}{A}+\gamma_{U H}\left(1-\varepsilon_{1}\right) K_{1} K_{5}$

$+\frac{\gamma_{U H} K_{2} \varepsilon_{1} K_{H} K_{5}}{A}+\frac{\gamma_{U H} K_{2} K_{H} \phi \tau_{1} \gamma_{U H}\left(1-\varepsilon_{1}\right)}{K_{14} K_{13} A}+\frac{\tau_{1} \omega_{1} K_{H} \varepsilon_{1} K_{3}}{A}+\frac{\tau_{1} \omega_{1} K_{H} \phi \tau_{1} \gamma_{U H}\left(1-\varepsilon_{1}\right)}{K_{15} K_{1}+A}+\gamma_{U H}\left(1-\varepsilon_{1}\right) K_{1} K_{5}$

$+\frac{\gamma_{U H} K_{2} K_{H} \varepsilon_{1} K_{5}}{A}+\frac{\gamma_{U H} K_{2} K_{H} \phi \tau_{1} \gamma_{U H}\left(1-\varepsilon_{1}\right)}{K_{4} K_{3} A}$

$=R_{H}+Q$ where

$Q=\frac{\beta}{K_{1} K_{3} K_{4} K_{5}}\left[\frac{\varepsilon_{1} K_{3} K_{5}}{A}\left(K_{4}+\omega_{1} K_{H}\right)+\frac{\varepsilon_{1} K_{2} K_{5} K_{H}}{A}\left(K_{4}+2 \gamma_{U H}\right)+\frac{\varepsilon_{1} \tau_{1} \omega_{1} K_{H} K_{3}}{A}\right.$

$\left.+3 \frac{\phi \tau_{1} \gamma_{U H}(1-\varepsilon)}{A}+\frac{\left(1-\varepsilon_{1}\right) 2 \gamma_{U H} K_{H}}{A K_{4} K_{3}}\left(K_{2} \phi \tau_{1}+K_{2} \phi \tau_{1}\right)+\left(1-\varepsilon_{1}\right) \gamma_{U H}\left(\frac{2 \tau_{1} \omega_{1} K_{H} \phi}{K_{5} K_{4} A}+2 K_{1} K_{5}+K_{1} K_{4} K_{5}\right)\right]$

Where $A=\left(1-\frac{\phi \tau_{1} w_{1} K_{H}}{K_{1} K_{4} K_{5}}-\frac{\phi \tau_{1} \gamma_{u H} K_{12} K_{H}}{K_{1} K_{4} K_{5} K_{3}}\right)$

Therefore, $1+P_{5} \lambda^{* * *}=R_{H}+Q$

$$
\lambda^{* *}=\frac{R_{H}+Q-1}{P_{5}}>0 \text { Whenever } R_{H}>1
$$

Therefore, there exist an endemic equilibrium

\subsection{TB MODEL ONLY}

$$
\begin{aligned}
& \frac{d S}{d t}=\pi-\lambda_{T} S-\mu \\
& \frac{d L_{T}}{d t}=\varepsilon_{2} \lambda_{T} S-K_{10} L_{T}+v T_{U}+\theta_{1} \rho F_{T}+r \alpha R_{T} \\
& \frac{d T_{U}}{d t}=\left(1-\varepsilon_{2}\right) \lambda_{H} S+K_{11} L_{T}+K_{12} L_{T}+\theta_{2} \rho F_{T}-K_{13} T_{U} \\
& \frac{d T_{D}}{d t}=\omega_{2} \kappa_{T} L_{T}+\omega_{3} \varphi \lambda_{T} L_{T}+\gamma_{U T} T_{U}+K_{14} F_{T}-K_{15} T_{D} \\
& \frac{d F_{T}}{d t}=K_{16} T_{D}+K_{17} R_{T}-K_{18} F_{T} \\
& \frac{d R_{T}}{d t}=q_{1} \tau_{2} T_{D}-K_{19} R_{T}+\sigma_{2} T_{D}
\end{aligned}
$$

Where $\lambda_{T}=\beta_{T} \frac{\left(L_{T}+\eta_{U} T_{U}+\eta_{d T} T_{D}+\eta_{R T} R_{T}+\eta_{F T} F_{T}\right)}{N}$

For this model, it can be shown that the region,

$D_{1}=\left\{\left(S+L_{T}+\eta_{U} T_{U}+\eta_{d T} T_{D}+\eta_{R T} R_{T}+\eta_{F T} F_{T}\right) \in R_{+}^{6}: N \leq \pi / \mu\right.$ 


\subsubsection{DERIVATION OF BASIC REPRODUCTION NUMBER (Ro) FOR TB ONLY}

Using the same approach in HIV only model, we have,

$$
F i=\left(\begin{array}{l}
F 1 \\
F 2 \\
F 3 \\
F 4 \\
F 5
\end{array}\right)=\left(\begin{array}{l}
\varepsilon_{2} \lambda_{T} S \\
\left(1-\varepsilon_{2}\right) \lambda_{T} S \\
0 \\
0 \\
0
\end{array}\right) \text { and } V i=\left(\begin{array}{l}
V 1 \\
V 2 \\
V 3 \\
V 4 \\
V 5
\end{array}\right)=\left(\begin{array}{l}
K_{10} L_{T}-v T_{U}-\theta_{1} \rho F_{T}-r \alpha R_{T} \\
-K_{11} L_{T}-\theta_{2} \rho F_{T}+K_{13} T_{U} \\
-\omega_{2} \kappa_{T} L_{T}-\gamma_{U T} T_{U}-K_{14} F_{T}+K_{15} T_{D} \\
-K_{16} T_{D}-K_{17} R_{T}+K_{18} F_{T} \\
-K_{19} T_{D}+K_{20} R_{T}
\end{array}\right)
$$

After taking partial derivative $\mathrm{F}$ and $\mathrm{V}$, we have

\begin{tabular}{|c|c|c|c|c|c|}
\hline \multirow{4}{*}{$F=$} & $\begin{array}{l}\varepsilon_{2} \beta_{T} \\
\left(1-\varepsilon_{2}\right) \beta_{T}\end{array}$ & $\begin{array}{l}\varepsilon_{2} \beta_{T} \eta_{U} \\
\left(1-\varepsilon_{2}\right) \beta_{T} \eta_{U}\end{array}$ & $\begin{array}{l}\varepsilon_{2} \beta_{T} \eta_{d T} \\
\left(1-\varepsilon_{2}\right) \beta_{T} \eta_{d T}\end{array}$ & $\begin{array}{l}\varepsilon_{2} \beta_{T} \eta_{R T} \\
\left(1-\varepsilon_{2}\right) \beta_{T} \eta_{R T}\end{array}$ & $\begin{array}{l}\varepsilon_{2} \beta_{T} \eta_{F T} \\
\left(1-\varepsilon_{2}\right) \beta_{T} \eta_{F T}\end{array}$ \\
\hline & 0 & 0 & 0 & 0 & 0 \\
\hline & 0 & 0 & 0 & 0 & 0 \\
\hline & 0 & 0 & 0 & 0 & 0 \\
\hline & $K_{10}$ & $-v$ & 0 & ${ }_{1} \rho$ & \\
\hline & $-K_{11}$ & $K_{13}$ & 0 & $-\theta_{2} \rho$ & \\
\hline$V=$ & $-\omega_{2} \kappa_{T}$ & $-\gamma_{U T}$ & $K_{15}$ & $-K_{14}$ & \\
\hline & 0 & 0 & $-K_{16}$ & $K_{18}$ & \\
\hline & 0 & 0 & $-K_{19}$ & ) & \\
\hline
\end{tabular}

$R_{T}=\left(\frac{B_{1}+B_{2}+B_{3}}{M}\right)$

Where

$$
\begin{aligned}
& M=\left[\begin{array}{l}
v K_{20} K_{18} K_{15} K_{11}+\gamma_{U T} \alpha r K_{19} K_{18} K_{11}-\gamma_{U T} \theta_{1} \rho K_{19} K_{17} K_{11}+\gamma_{U T} \theta_{1} \rho K_{20} K_{16} K_{11} \\
-v K_{19} K_{17} K_{14} K_{11}+\gamma_{U T} \theta_{2} \rho K_{19} K_{17} K_{10}+v \omega_{2} \theta_{2} \kappa_{T} \rho K_{19} K_{17}+K_{20} K_{16} K_{14} K_{13} K_{10} \\
-K_{20} K_{18} K_{15} K_{13} K_{10}+\omega_{2} \theta_{1} \kappa_{T} \rho K_{19} K_{17} K_{13}-\gamma_{U T} \theta_{2} \rho K_{20} K_{16} K_{10}+v K_{20} K_{16} K_{14} K_{11} \\
-\omega_{2} \kappa_{T} r \alpha K_{19} K_{18} K_{13}-v \omega_{2} \theta_{2} \kappa_{T} \rho K_{20} K_{16}-\omega_{2} \theta_{1} \kappa_{T} \rho K_{20} K_{16} K_{13}-K_{19} K_{17} K_{14} K_{13} K_{10}
\end{array}\right] \\
& B_{1}=\left(\beta _ { T } \left(r \alpha K_{19} K_{18} \gamma_{U T}+K_{20} K_{18} K_{15} v+K_{20} K_{14} K_{16} v-K_{19} K_{16} K_{14} v+\gamma_{U T} \theta_{1} \rho K_{19} K_{17}-\gamma_{U T} \theta_{1} \rho K_{20} K_{16}\right.\right. \\
& -\varepsilon_{2} \eta_{U} K_{19} K_{17} K_{14} K_{11}+\varepsilon_{2} \eta_{d T} K_{18} K_{20} \gamma_{U T}+\varepsilon_{2} \eta_{R T} K_{11} K_{16} K_{20} \gamma_{U T}-\varepsilon_{2} \eta_{R T} K_{11} K_{19} K_{17} \gamma_{U T}+\varepsilon_{2} \eta_{F T} K_{11} K_{18} K_{19} \gamma_{U T} \\
& \left.\left.-\varepsilon_{2} r \alpha K_{19} K_{18} \gamma_{U T}-\varepsilon_{2} \eta_{U} K_{10} K_{16} K_{14} K_{20}+\varepsilon_{2} \eta_{U} K_{19} K_{17} K_{14} K_{10}-\varepsilon_{2} \eta_{U} K_{15} K_{20} K_{10} K_{18}-\varepsilon_{2} \eta_{d T} K_{10} K_{18} K_{20} \gamma_{U T}\right)\right)
\end{aligned}
$$

$B_{2}=\left(\beta_{T}\left(\varepsilon_{2} \eta_{R T} K_{10} K_{19} K_{17} \gamma_{U T}-\varepsilon_{2} \eta_{R T} K_{10} K_{15} K_{20} \gamma_{U T}-\varepsilon_{2} \rho K_{20} K_{16} \theta_{2} \gamma_{U T}+\varepsilon_{2} \rho K_{19} K_{17} \theta_{2} \gamma_{U T}\right.\right.$

$+\varepsilon_{2} \eta_{U} K_{11} K_{15} K_{18} K_{20}+\varepsilon_{2} \eta_{U} K_{11} K_{14} K_{16} K_{20}+\omega_{2} \eta_{U} \kappa_{T} \theta_{1} K_{16} K_{20}-\omega_{2} \eta_{U} \kappa_{T} \theta_{1} K_{19} K_{17}+\omega_{2} \eta_{R T} \kappa_{T} v K_{16} K_{20}$

$-\omega_{2} \eta_{R T} \kappa_{T} v K_{17} K_{19}+\omega_{2} \eta_{d T} \kappa_{T} v K_{18} K_{20}+\omega_{2} \eta_{F T} \kappa_{T} v K_{18} K_{19}-\varepsilon_{2} \omega_{2} \eta_{U} \kappa_{T} \theta_{1} K_{16} K_{20}+\varepsilon_{2} \omega_{2} \eta_{U} \kappa_{T} \theta_{1} K_{19} K_{17}$

$-\varepsilon_{2} \omega_{2} \eta_{d T} \kappa_{T} v K_{18} K_{20}-\varepsilon_{2} \omega_{2} \eta_{R T} \kappa_{T} v K_{16} K_{20}+\varepsilon_{2} \omega_{2} \eta_{R T} \kappa_{T} v K_{17} K_{19}-\varepsilon_{2} \omega_{2} \eta_{F T} \kappa_{T} v K_{18} K_{19}$

$\left.\left.+\varepsilon_{2} \omega_{2} \eta_{d T} \kappa_{T} K_{13} K_{18} K_{20}\right)\right)$

$B_{3}=\left(\beta_{T}\left(\varepsilon_{2} \omega_{2} \eta_{R T} \kappa_{T} K_{13} K_{16} K_{20}-\varepsilon_{2} \omega_{2} \eta_{R T} \kappa_{T} K_{13} K_{19} K_{13}+\varepsilon_{2} \omega_{2} \eta_{F T} \kappa_{T} K_{13} K_{18} K_{19}-r \alpha \omega_{2} \eta_{U} \kappa_{T} K_{19} K_{18}\right.\right.$

$-\varepsilon_{2} \nu K_{15} K_{18} K_{20}-\varepsilon_{2} \nu K_{14} K_{16} K_{20}+\varepsilon_{2} \nu K_{14} K_{17} K_{19}+\varepsilon_{2} K_{20} K_{16} \theta_{1} \gamma_{U T}-\varepsilon_{2} K_{19} K_{17} \theta_{1} \gamma_{U T}+\varepsilon_{2} K_{20} K_{16} K_{13} K_{14}$

$-\varepsilon_{2} K_{19} K_{17} K_{13} K_{14}+\varepsilon_{2} K_{20} K_{15} K_{13} K_{18}+\eta_{U} K_{20} K_{16} K_{10} K_{14}-\eta_{U} K_{19} K_{17} K_{10} K_{14}+\eta_{U} K_{20} K_{14} K_{10} K_{18}$

$+\eta_{R T} K_{10} K_{16} K_{20} \gamma_{U T}-\eta_{R T} K_{1} K_{17} K_{19} \gamma_{U T}+\eta_{d T} K_{10} K_{18} K_{20} \gamma_{U T}+\eta_{F T} K_{10} K_{18} K_{19} \gamma_{U T}+\varepsilon_{2} r \alpha \omega_{2} \eta_{U} \kappa_{T} K_{19} K_{18}$

$\left.\left.+\eta_{U} \varepsilon_{2} \rho K_{20} K_{16} \theta_{2} \omega_{2} \kappa_{T}-\eta_{U} \varepsilon_{2} \rho K_{19} K_{17} \theta_{2} \omega_{2} \kappa_{T}\right)\right)$ 
The threshold quantity $R_{T}$ is the basic reproduction number of the normalized model system (35) for HIV infection in a population. It measures the average number of new secondary infections generated by a single infected individual in his or her infectious period in a population [1].

\subsubsection{DISEASE FREE EQUILIBRIUM}

For critical points, we set

$\frac{d S}{d t}=\frac{d L_{T}}{d t}=\frac{d T_{U}}{d t}=\frac{d T_{D}}{d t}=\frac{d F_{T}}{d t}=\frac{d R_{T}}{d t}=0$

At this free equilibrium, it is assumed that there is no infection, Hence the (DFE) is given as

$\varepsilon_{0}=\left(S, L_{T}, T_{U}, T_{D}, F_{T}, R_{T}\right)=\left(\frac{\pi}{\mu}, 0,0,0,0,0\right)$

\subsubsection{GLOBAL STABILITY OF DISEASE FREE EQUILIBRIUM (TB)}

Theorem3.5: The disease free-equilibrium of the system (35) is globally asymptotically stable whenever $R_{T}<1$ and unstable if $R_{T}>1$.

Proof: It follows that $S=N^{*}-L_{T}-T_{u}-T_{D}-F_{T}-R_{T}$ at steady state. The proof is based on using the comparison method.[21]
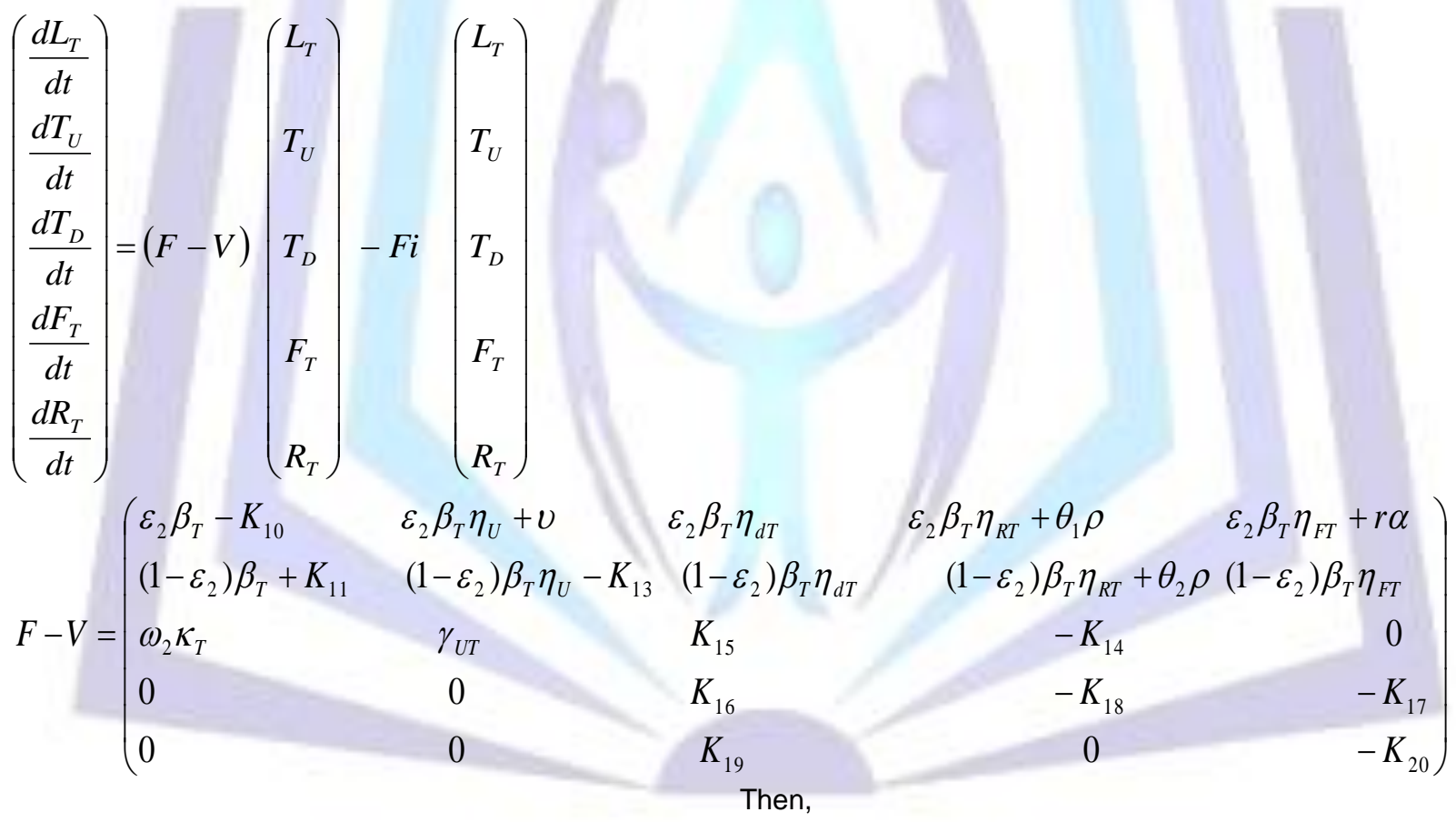

According to $[6,31]$, all eigenvalues of the matrix

$(\mathrm{F}-\mathrm{V})$ have negative real parts.

Thus, we have

$\left[\begin{array}{l}\left(\left(\varepsilon_{2} \beta_{T}-K_{10}\right)-\lambda\right)\left(\left(1-\varepsilon_{2}\right) \beta_{T} \eta_{U}-K_{13}-\lambda\right)\left(K_{15}-\lambda\right)\left(\left(-K_{18}-\lambda\right)\left(-K_{20}-\lambda\right)\right)-\left(\left(\varepsilon_{2} \beta_{T} \eta_{U}+v\right)\right. \\ \left.\left(\left(1-\varepsilon_{2}\right) \beta_{T}+K_{11}\right)\left(K_{15}-\lambda\right)\left(-K_{18}-\lambda\right)\left(-K_{20}-\lambda\right)\right)+\left(\left(\varepsilon_{2} \beta_{T} \eta_{d T}\right)\left(\left(1-\varepsilon_{2}\right) \beta_{T}+K_{11}\right)\left(\gamma_{U T}\right)\right. \\ \left.\left(-K_{18}-\lambda\right)\left(-K_{20}-\lambda\right)\right)-\left(\left(\varepsilon_{2} \beta_{T} \eta_{R T}+\theta_{2} \rho\right)\left(\left(1-\varepsilon_{2}\right) \beta_{T}+K_{11}\right)\left(\gamma_{U T}\right)\left(-K_{20}-\lambda\right)\left(K_{16}\right)+K_{17} K_{19}\right) \\ +\left(\left(\varepsilon_{2} \beta_{T} \eta_{F T}+r \alpha\right)\left(\left(1-\varepsilon_{2}\right) \beta_{T}+K_{11}\right)\left(\gamma_{U T}\right)\left(-K_{18}-\lambda\right)\left(K_{19}\right)\right.\end{array}\right]$

Thus from equation (45), the characteristic equation is given by 
$\lambda^{5}+a_{5} \lambda^{4}+a_{4} \lambda^{3}+a_{3} \lambda^{2}+a_{2} \lambda+a_{1}=0$

Thus according to Routh Hurwitz Criteria for order five (5),

$a_{5}>0 \quad a_{4}>0 \quad a_{3}>0 \quad a_{2}>0 \quad a_{1}>0$

Then for $a_{1}>0$ we have

$-\beta_{T}\left(B_{1}+B_{2}+B_{3}\right)>-M$

Hence

$\frac{\beta_{T}\left(B_{1}+B_{2}+B_{3}\right)}{M}<1$

Implying that

$R_{T}<1$.

Hence, we have established that the disease free equilibrium is globally asymptotically stable whenever $R_{T}<1$ and unstable $R_{T}>1$.

\subsubsection{EXISTENCE OF ENDEMIC FOR TB MODEL ONLY}

Where $\varepsilon_{0}^{* *}=\left(S^{* *}, L_{T}^{* *}, T_{U}^{* *}, T_{D}^{* *}, F_{T}^{* *}, R_{T}^{* *}\right)$ are the endemic equilibrium points.

Equation (35) becomes

$\frac{d S}{d t}=\pi-\lambda_{T} S-\mu S$

$\frac{d L_{T}}{d t}=\varepsilon_{2} \lambda_{T} S-K_{10} L_{T}$

$\frac{d T_{U}}{d t}=\left(1-\varepsilon_{2}\right) \lambda_{T} S+K_{11} L_{T}-K_{13} T_{U}$

$\frac{d T_{D}}{d t}=\omega_{2} \kappa_{T} L_{T}+\gamma_{U T} T_{U}-K_{15} T_{D}$

$\frac{d R_{T}}{d t}=K_{19} T_{D}-\mu R_{T}+\sigma_{2} T_{D}$

For a special case of TB-only model, when $q_{1}=1$ and $v_{1}, \theta_{1}, \theta_{2} \varphi$ and $\alpha$ are very small (negligible). Then, solving at steady state;

$S^{* *}=\frac{\pi}{\lambda_{T}^{* *}+\mu}$

$L_{T}^{* *}=\frac{\varepsilon_{2} \lambda_{T}^{* * *} S^{* *}}{K_{10}}$

$T_{U}^{* *}=\frac{\left(1-\varepsilon_{2}\right) \lambda_{T}^{* * *} S^{* * *}+K_{11} \kappa_{T} L_{T}^{* * *}}{K_{13}}$

$T_{D}^{* *}=\frac{\omega_{2} \kappa_{T} L_{T}^{* *}+\gamma_{U T} T_{U}^{* *}}{K_{15}}$

$R_{T}^{* *}=\frac{K_{19} T_{D}^{* *}}{\mu}$ 
The expression for $\lambda_{T}$ at the endemic steady-state, denoted by $\lambda^{* *}$ is given by

$$
\begin{aligned}
& \lambda_{T}^{* *}=\frac{\beta_{T}\left\lfloor\eta_{U} T_{U}^{* *}+\eta_{d T} T_{D}^{* * *}+\eta_{R T} R_{T}^{* * *}+\eta_{F T} F_{T}^{* *}+L_{T}^{* *}\right\rfloor}{N^{* *}} \\
& L_{T}^{* *}=\frac{\varepsilon_{2} \lambda_{T}^{* *} S^{* *}}{K_{10}} \\
& T_{u}^{* *}=\frac{\left(1-\varepsilon_{2}\right) \lambda_{T}^{* * *} S^{* *}}{K_{13}}+\frac{K_{11} \kappa_{T} \varepsilon_{2} \lambda_{T}^{* *} S^{* *}}{K_{13} K_{10}}=P_{1} \lambda^{* *} S^{* *} \\
& T_{D}^{* *}=\frac{\omega_{2} \kappa_{T} \varepsilon_{2} \lambda_{T}^{* *} S^{* *}}{K_{10} K_{15}}+\frac{\gamma_{U T} P_{1} \lambda^{* *} S^{* *}}{K_{15}}=P_{2} \lambda^{* *} S^{* *} \\
& R_{T}^{* *}=\frac{K_{19}}{\mu}\left[\frac{\omega_{2} \kappa_{T} \varepsilon_{2} \lambda_{T}^{* * *} S^{* *}}{K_{10} K_{15}}+\frac{\gamma_{U T} P_{1} \lambda^{* *} S^{* *}}{K_{15}}\right]=P_{3} \lambda^{* * *} S^{* *} \\
& P_{1}=\frac{\left(1-\varepsilon_{2}\right)}{K_{13}}-\frac{K_{11} \kappa_{T} \varepsilon_{2}}{K_{10} K_{13}} \\
& P_{2}=\frac{\omega_{2} \kappa_{T} \varepsilon_{2}}{K_{10} K_{15}}+\frac{\gamma_{U T}}{K_{15}}\left[\frac{\left(1-\varepsilon_{2}\right)}{K_{13}}-\frac{K_{11} \kappa_{T} \varepsilon_{2}}{K_{10} K_{13}}\right] \\
& P_{3}=\frac{K_{19}}{\mu}\left[\frac{\omega_{2} \kappa_{T} \varepsilon_{2}}{K_{10} K_{15}}+\frac{\gamma_{U T}}{K_{15}}\left(\frac{\left(1-\varepsilon_{2}\right)}{K_{13}}-\frac{K_{11} \kappa_{T} \varepsilon_{2}}{K_{10} K_{13}}\right)\right]
\end{aligned}
$$

Substituting the expression in (47.12) into (47.11)

$$
\lambda^{* *}\left[S^{* *}+\frac{\varepsilon_{2} \lambda_{T}^{* *} S^{* *}}{K_{10}}+P_{1} \lambda^{* *} S^{* * *}+P_{2} \lambda^{* *} S^{* *}+P_{3} \lambda^{* *} S^{* *}\right]=\beta \lambda_{T}^{* * *} S^{* *}\left[\eta_{U} P_{1}+\eta_{d T} P_{2}+\eta_{R T} P_{3}\right]
$$

Divide each term in (47.14) by $\lambda_{T}^{* *} S^{* *}$

$1+P_{4} \lambda^{* *}=\beta\left[\eta_{U} P_{1}+\eta_{d T} P_{2}+\eta_{R T} P_{3}\right]$

Where $P_{4}=\frac{\varepsilon_{2}}{K_{10}}+P_{1}+P_{2}+P_{3} \geq 0$

So that

$$
\begin{aligned}
1+P_{4} \lambda^{* *} & =\frac{\beta_{T}}{K_{10} K_{13} K_{15}}\left[\eta_{U}\left(1-\varepsilon_{2}\right) K_{10} K_{15}+\eta_{U} K_{11} \kappa_{T} \varepsilon_{2} K_{13}+\eta_{d T} \omega_{2} \kappa_{T} \varepsilon_{2} K_{13}\right. \\
& +\eta_{d T} \gamma_{U T}\left[\left(1-\varepsilon_{2}\right) K_{10}+K_{11} \kappa_{T} \varepsilon_{2}\right]+\eta_{R T} \frac{K_{19}}{\mu} \omega_{2} \kappa_{T} \varepsilon_{2} K_{13}+\eta_{R T} \frac{K_{19}}{\mu} \gamma_{U T} \\
& {\left[\left(1-\varepsilon_{2}\right) K_{10}+K_{11} \kappa_{T} \varepsilon_{2}\right]=R_{T}+Q }
\end{aligned}
$$

Where 


$$
\begin{aligned}
& Q=\frac{\beta_{T}}{K_{11} K_{12} K_{13}}\left[\left(1-\varepsilon_{2}\right) K_{11}\left[\eta_{U} K_{13}+\eta_{d T} \gamma_{U T}+\eta_{R T} \frac{\left(\sigma_{2}+\tau_{2}\right)}{\mu}\right]+\right. \\
& \left.\quad\left(1-\omega_{2}\right) \kappa_{T} \varepsilon_{2}\left[\eta_{U} K_{12}+\eta_{d T} \gamma_{U T}+\eta_{R T} \gamma_{U T} \frac{\left(\sigma_{2}+\tau_{2}\right)}{\mu}\right]+\omega_{2} \kappa_{T} \varepsilon_{2} K_{12}\left[\eta_{d T}+\eta_{R T} \frac{\left(\sigma_{2}+\tau_{2}\right)}{\mu}\right]\right]
\end{aligned}
$$

Therefore $1+P_{4} \lambda_{T}^{* *}=R_{T}+Q$

$\lambda_{T}^{* * *}=\frac{R_{T}+Q-1}{P_{4}}>0$, whenever $R_{T}>1$

Therefore, there exist an endemic equilibrium whenever $R_{T}>1$

\subsection{ANALYSIS OF THE FULL MODEL}

Consider the Co-infection model (23) of HIV-TB

\subsubsection{DISEASE FREE EQUILIBRIUM}

For critical points, we set

$\frac{d S}{d t}=\frac{d L_{H}}{d t}=\frac{d H_{U}}{d t}=\frac{d H_{D}}{d t}=\frac{d H_{W}}{d t}=\frac{d L_{T H}}{d t}=\frac{d A_{T H}}{d t}=\frac{d L_{H T}}{d t}=\frac{d A_{H T}}{d t}=\frac{d L_{T}}{d t} \frac{d T_{U}}{d t}=\frac{d T_{D}}{d t}=\frac{d F_{T}}{d t}=\frac{d R_{T}}{d t}=\frac{d R_{T H}}{d t}=0$

At disease free equilibrium, it is assumed that there is no infection. Then, the disease free equilibrium of the full model is given by

$$
\begin{aligned}
& \varepsilon_{1}=\left(S^{*}, L_{H}{ }^{*}, H_{U}{ }^{*}, H_{D}{ }^{*}, H_{W}{ }^{*}, L_{T H}{ }^{*}, A_{T H}{ }^{*}, L_{H T}{ }^{*}, A_{H T}{ }^{*}, L_{T}{ }^{*}, T_{U}{ }^{*}, T_{D}{ }^{*}, F^{*}, R_{T}{ }^{*}, R_{T H}{ }^{*}\right) \\
& =\left(\frac{\pi}{\mu}, 0,0,0,0,0,0,0,0,0,0,0,0,0\right)
\end{aligned}
$$

\subsubsection{DERIVATION OF BASIC REPRODUCTION NUMBER $\left(\mathbf{R}_{0}\right)$ FOR HIV-TB CO-INFECTION}

The associated matrices $\mathrm{F}$ and $\mathrm{V}$, are given, respectively, by

$$
F=\left[\begin{array}{ll}
F_{1} & F_{2} \\
F_{3} & F_{4}
\end{array}\right]
$$

Where,

$$
F_{1}=\left(\begin{array}{lllllll}
\varepsilon_{1} \beta_{H} & \varepsilon_{1} \beta_{H} \eta_{U} & \varepsilon_{1} \beta_{H} \eta_{d H} & \varepsilon_{1} \beta_{H} \eta_{W} & \varepsilon_{1} \beta_{T} & \varepsilon_{1} \beta_{T} \eta_{T} \varepsilon_{1} \beta_{H} & \\
\left(1-\varepsilon_{1}\right) \beta_{H} & \left(1-\varepsilon_{1}\right) \beta_{H} \eta_{U} & \left(1-\varepsilon_{1}\right) \beta_{H} \eta_{d H} & \left(1-\varepsilon_{1}\right) \beta_{H} \eta_{W} & \left(1-\varepsilon_{1}\right) \beta_{T} & \left(1-\varepsilon_{1}\right) \beta_{T} \eta_{T} & \left(1-\varepsilon_{1}\right) \beta_{H} \\
0 & 0 & 0 & 0 & 0 & 0 & 0 \\
0 & 0 & 0 & 0 & 0 & 0 & 0 \\
\beta_{H} & \beta_{H} \eta_{U} & \beta_{H} \eta_{d H} & \beta_{H} \eta_{W} & \beta_{T} & \beta_{T} \eta_{T} & \beta_{H} \\
0 & 0 & 0 & 0 & 0 & 0 & 0 \\
\beta_{H} & \beta_{H} \eta_{U} & \beta_{H} \eta_{d H} & \beta_{H} \eta_{W} & \beta_{T} & \beta_{T} \eta_{T} & \beta_{H}
\end{array}\right)
$$




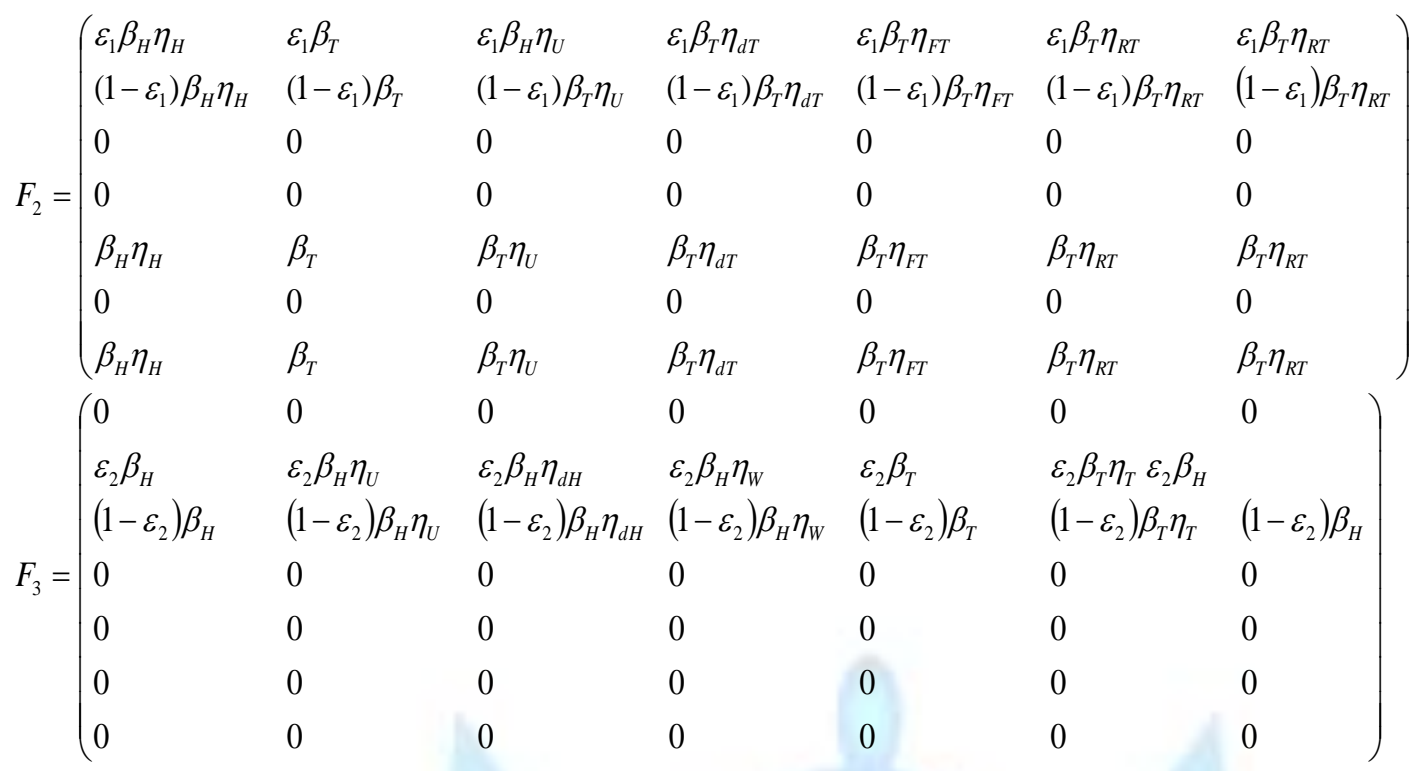

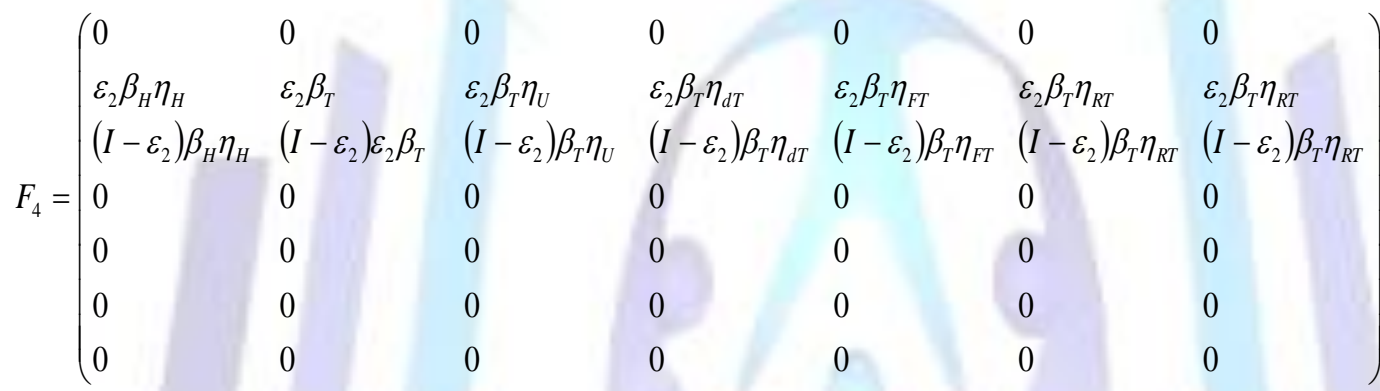

$$
\text { And, } V=\left[\begin{array}{ll}
V_{1} & V_{2} \\
V_{3} & V_{4}
\end{array}\right]
$$

Where,

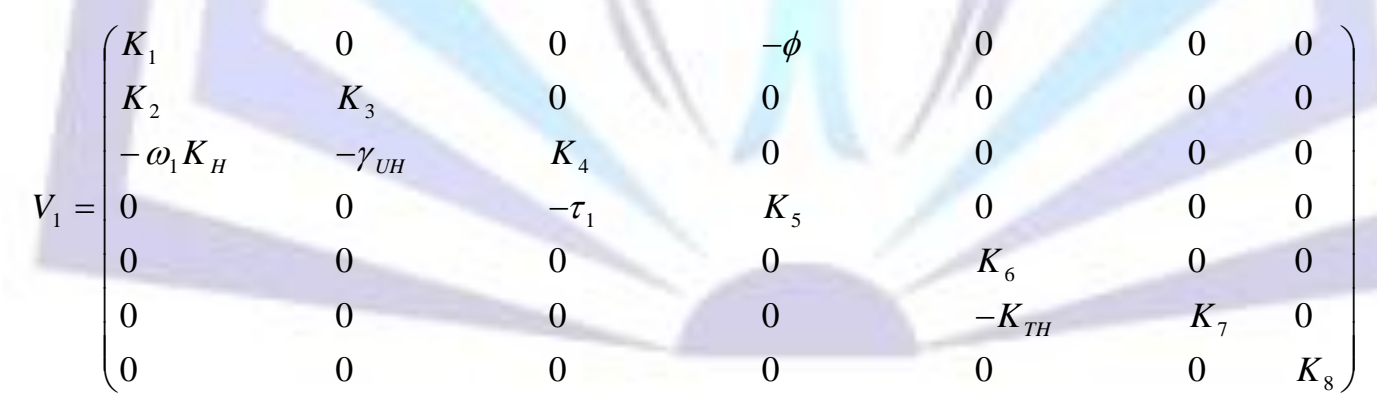

$$
V_{2}=\left(\begin{array}{lllllll}
0 & 0 & 0 & 0 & 0 & 0 & 0 \\
0 & 0 & 0 & 0 & 0 & 0 & 0 \\
0 & 0 & 0 & 0 & 0 & 0 & 0 \\
0 & 0 & 0 & 0 & 0 & 0 & 0 \\
0 & 0 & 0 & 0 & 0 & 0 & -\alpha T \\
0 & 0 & 0 & 0 & 0 & 0 & 0 \\
0 & 0 & 0 & 0 & 0 & 0 & 0
\end{array}\right)
$$




$$
\begin{aligned}
& V_{3}=\left(\begin{array}{lllllll}
0 & 0 & 0 & 0 & 0 & 0 & 0 \\
0 & 0 & 0 & 0 & 0 & 0 & 0 \\
0 & 0 & 0 & 0 & 0 & 0 & 0 \\
0 & 0 & 0 & 0 & 0 & 0 & 0 \\
0 & 0 & 0 & 0 & 0 & 0 & 0 \\
0 & 0 & 0 & 0 & 0 & 0 & 0 \\
0 & 0 & 0 & 0 & 0 & 0 & 0 \\
0 & 0 & 0 & 0 & 0 & -\sigma_{1} & 0
\end{array}\right) \\
& V_{4}=\left(\begin{array}{lllllll}
K_{9} & 0 & 0 & 0 & 0 & 0 & 0 \\
0 & K_{10} & -v & 0 & -\theta_{1} \rho & -r \alpha & 0 \\
0 & K_{11} & K_{13} & 0 & -\theta_{2} \rho & 0 & 0 \\
0 & -\omega_{2} & -\gamma_{U T} & K_{14} & K_{15} & 0 & 0 \\
0 & 0 & 0 & K_{16} & K_{17} & K_{18} & 0 \\
0 & 0 & 0 & K_{19} & 0 & K_{20} & 0 \\
0 & 0 & 0 & 0 & 0 & 0 & K_{21}
\end{array}\right) \\
& F \cdot V^{-1}=\left[\begin{array}{ll}
F_{1} \cdot V_{1}^{-1} & F_{2} \cdot V_{2}^{-1} \\
F_{3} \cdot V_{3}^{-1} & F_{4} \cdot V_{4}^{-1}
\end{array}\right] \\
& R_{1}=F_{1} \cdot V_{1}^{-1} \\
& R_{1}=\frac{\beta_{H} \varepsilon_{1}}{A}\left[K_{5} K_{4} K_{3}+\eta_{U} \phi \tau_{1} \gamma_{U H}+\eta_{d H} \phi \tau_{1} K_{3}+\eta_{W} \phi K_{4} K_{3}\right] \\
& \left.+\frac{\beta_{H}\left(1-\varepsilon_{1}\right)}{A}\left[-K_{5} K_{4} K_{2}-\eta_{U}\left(K_{5} K_{4} K_{1}-\phi \tau_{1} \omega_{1} \kappa_{H}\right)-\eta_{d H} \phi \tau_{1} K_{2}+\eta_{W} \phi K_{4} K_{2}\right]+\frac{\beta_{T}}{K_{8}}\right\} \\
& A=K_{5} K_{4} K_{3} K_{1}-\tau_{1} \phi \omega_{1} K_{4} K_{3}+\tau_{1} \phi \gamma_{U H} K_{2} \\
& R_{2}=F_{4} V_{4}^{-1} \\
& R_{2}=\frac{P_{1}+P_{2}+P_{3}+P_{4}+P_{5}+P_{6}+P_{7}+P_{8}+P_{9}+P_{10}}{B} \\
& P_{1}=\frac{-\left(\varepsilon_{2} \beta_{T}\left(K_{20} K_{16} \rho \gamma_{U T} \theta_{2}-K_{20} K_{16} K_{13} K_{15}+K_{19} K_{15} K_{13} K_{18}+K_{14} K_{20} K_{13} K_{17}-K_{19} K_{18} \rho \gamma_{U T} \theta_{2}\right)\right)}{B} \\
& P_{2}=\frac{\left(\varepsilon_{2} \beta_{T} \delta_{U}\left(K_{11} K_{14} K_{17} K_{20}-K_{11} K_{15} K_{16} K_{20}+K_{11} K_{15} K_{18} K_{19}+K_{20} K_{16} \rho \omega_{2} \theta_{2}-K_{19} K_{18} \rho \omega_{2} \theta_{2}\right)\right)}{B} \\
& P_{3}=\frac{\left(\varepsilon_{2} \beta_{T} \beta \delta_{d T} K_{17} K_{20}\left(K_{11} \gamma_{U T}-K_{13} \omega_{2}\right)\right)}{B} \\
& P_{4}=\frac{\left(\varepsilon_{2} \beta_{T} \delta_{F T}\left(K_{16} K_{20}-K_{18} K_{19}\right)\left(K_{11} \gamma_{U T}-K_{13} \omega_{2}\right)\right)}{B} \\
& P_{5}=\frac{-\left(\varepsilon_{2} \beta_{T} \delta_{R T} K_{17} K_{19}\left(K_{11} \gamma_{U T}-K_{13} \omega_{2}\right)\right)}{B}
\end{aligned}
$$


$P_{6}=\frac{\left(\begin{array}{l}\left(1-\varepsilon_{2}\right) \beta_{T}\left(-v K_{14} K_{17} K_{20}+v_{15} K_{16} K_{20}-v K_{15} K_{18} K_{19}+K_{20} K_{16} \rho \gamma_{U T} \theta_{1}+r \alpha K_{19} K_{17} \gamma_{U T}\right. \\ -K_{19} K_{18} \rho \gamma_{U T} \theta_{1}\end{array}\right)}{B}$

$P_{7}=\frac{\begin{array}{l}\left(1-\varepsilon_{2}\right) \beta_{T} \delta_{U}\left(K_{14} K_{20} K_{10} K_{17}-K_{20} K_{16} K_{18} K_{15}+K_{19} K_{15} K_{10} K_{18}-K_{19} K_{18} \rho \omega_{2} \theta_{1}+K_{20} K_{16} \rho \theta_{1}\right. \\ B\end{array}}{B}$

$P_{8}=\frac{-\left(\left(1-\varepsilon_{2}\right) \beta_{T} \delta_{d T} K_{17} K_{19}\left(v \omega_{2}+K_{10} \gamma_{U T}\right)\right.}{B}$

$P_{9}=\frac{\left(\left(1-\varepsilon_{2}\right) \beta_{T} \delta_{F T}\left(K_{16} K_{20}-K_{18} K_{19}\left(v \omega_{2}+K_{10} \gamma_{U T}\right)\right)\right.}{B}$

$P_{10}=\frac{\left(\left(1-\varepsilon_{2}\right) \beta_{T} \delta_{R T} K_{17} K_{19}\left(v \omega_{2}+K_{10} \gamma_{U T}\right)\right)}{B}$

Where;

$B=\left(v K_{11} K_{20} K_{16} K_{15}-v K_{11} K_{20} K_{17}-v K_{11} K_{19} K_{15} K_{18}-v K_{20} K_{16} \rho \omega_{2} \theta_{2}+v K_{14} \rho \omega_{2} \theta_{2} K_{18}+\right.$

$K_{11} K_{20} \rho \omega_{2} \theta_{2} K_{18}+K_{11} K_{20} K_{16} \rho \gamma_{U T} \theta_{1}+K_{11} K_{19} r \alpha \gamma_{U T} K_{17}-K_{11} K_{19} \rho \gamma_{U T} \theta_{2}-K_{19} r \alpha \omega_{2}$

$\left.-K_{20} K_{16} \gamma_{U T} K_{10} \theta_{2}+K_{19} \omega_{2} \theta_{1} \rho K_{12} K_{18}+K_{19} \rho \gamma_{U T} K_{10} \theta_{2} K_{18}\right)$

Hence it is shown that the associated Reproduction number is given by

$R_{C}=\max \left[R_{1}, R_{2}\right]$.

\subsection{NUMERICAL SIMULATIONS}

Table 1. Parameter Values used in Numerical Simulations

\section{Parameters}

$\pi$

$\mu$

$\tau_{1, \tau_{2}}$

$\varepsilon_{1}, \varepsilon_{2}$

$\kappa_{H}, \kappa_{T}$

$\omega_{1}, \omega_{2}$

$\gamma_{U H}, \gamma_{U T}$

$\phi$

$\beta_{H}, \beta_{T}$

$\delta_{U H}, \delta_{d H}$

$\eta_{U}, \eta_{d H} \eta_{W}$

$\theta_{1}, \theta_{2}$

$\varphi$
Value

2000

0.02

$0.20619,0.20619$

$0.7,0.7$

$0.2522,0.2522$

$0.2,0.2$

$0.2,0.2$

0.7

$0.1,0.1$

$0.3,0.1$

$0.001,0.001,0.001$

$0.1,0.1$

0.85
Source

[23]

[4]

Assumed

[26]

[10]

[26]

Assumed

[32]

[9]

[9]

[26]

Assumed 
$\kappa_{H T}, \kappa_{T H}$

$$
\omega_{2}, \omega_{3}
$$

$v$

$\alpha$

r

$\rho$

$\delta_{U T}, \delta_{d T}, \delta_{F}, \delta_{R T}$

$\sigma_{1}, \sigma_{2}$

$q_{1}$

$\eta_{U}, \eta_{d T}, \eta_{F T}, \eta_{R T}$
$0.2522,0.2522$

$0.7,0.7$

0.2

5

0.8

0.1

$0.3,0.1$

0.2

0.7

$0.001,0.001,0.001,0.001$
[10]

[23]

[11]

Assumed

Assumed

[10]

[32]

The new fifteen (15) compartmental non linear differential equations were designed to gain more insight on the effect of epidemiological features on the dynamical spread of HIV-TB co-infection. Numerical simulation of the model was carried out by maple software, using differential transformation method as to be able to determine the dynamical spread pattern of the disease in the community. Graphs and tables feature prominently in the discussion of results.

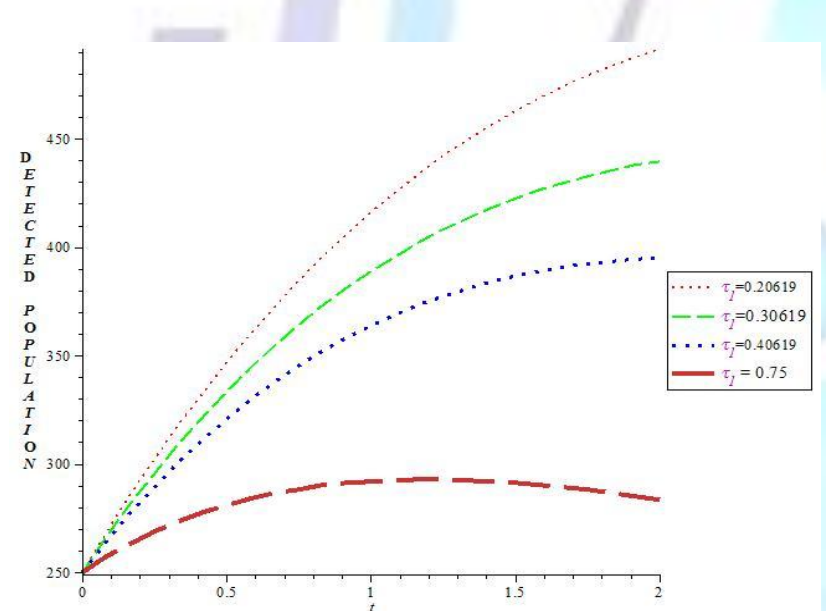

Fig. 1

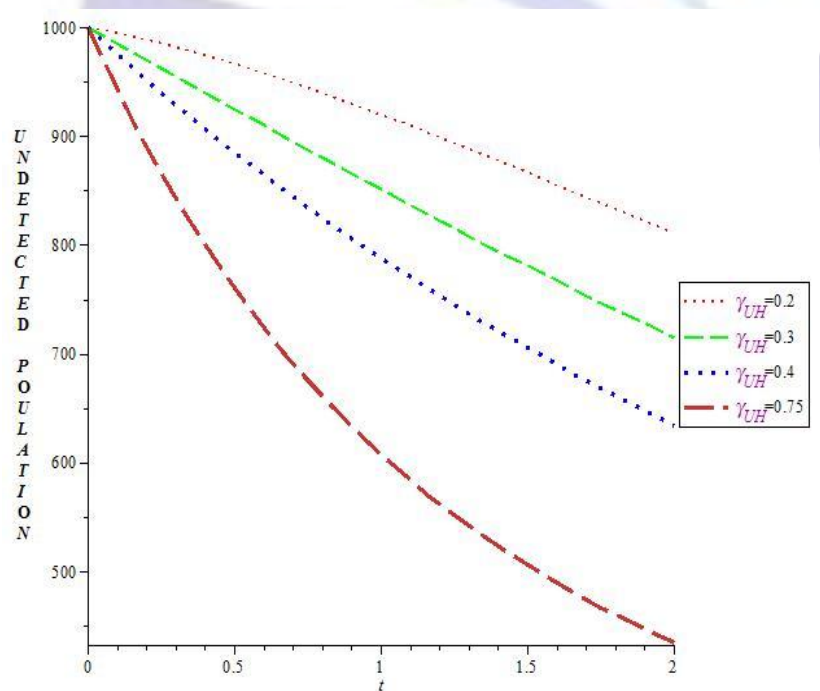

Fig. 3

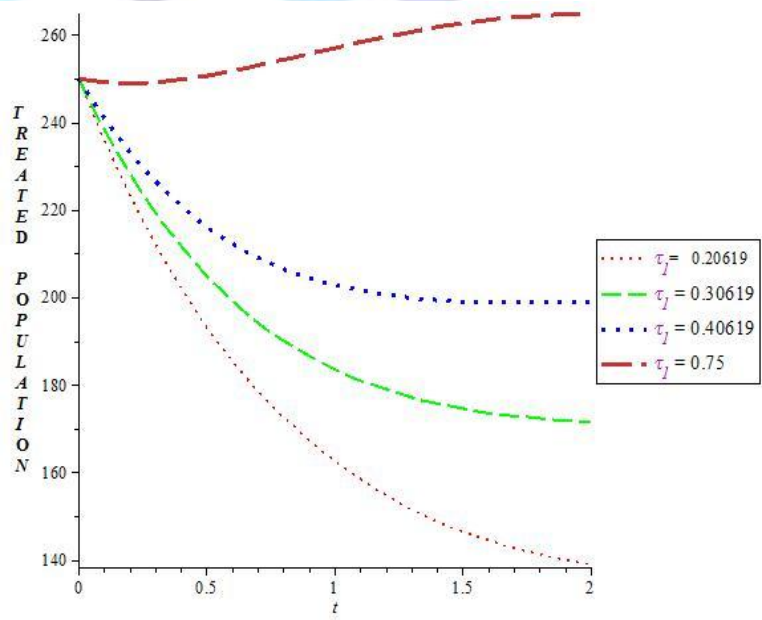

Fig. 2

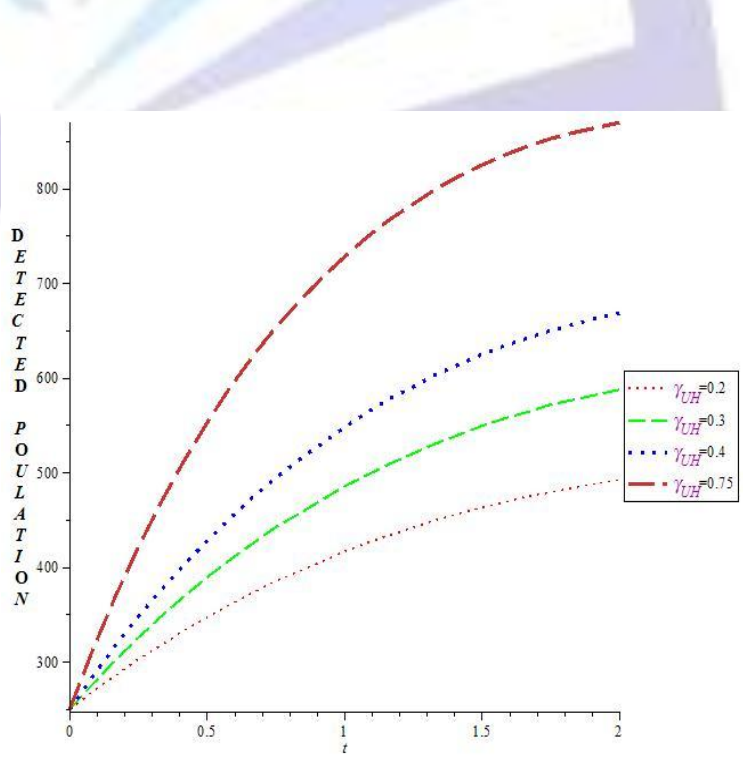

Fig. 4 


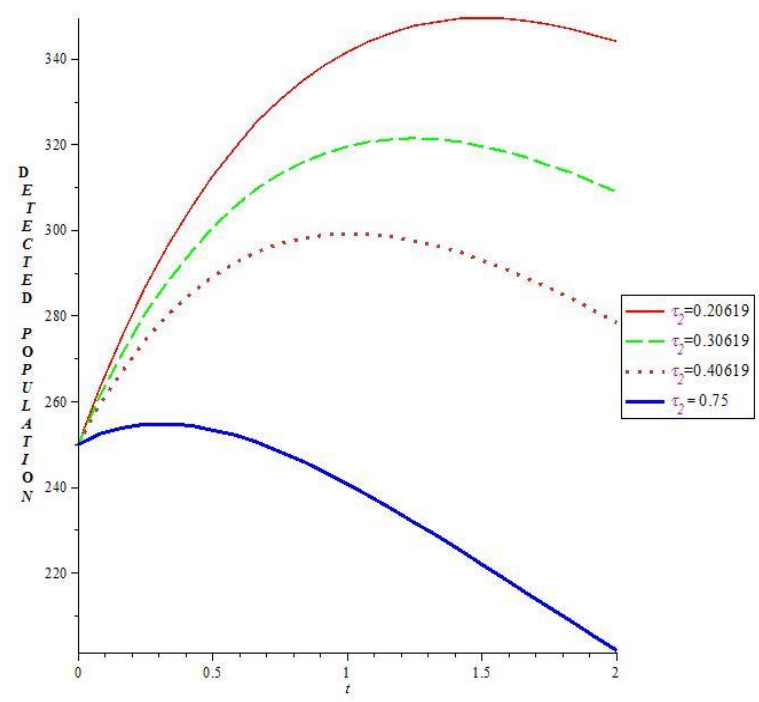

Fig. 5

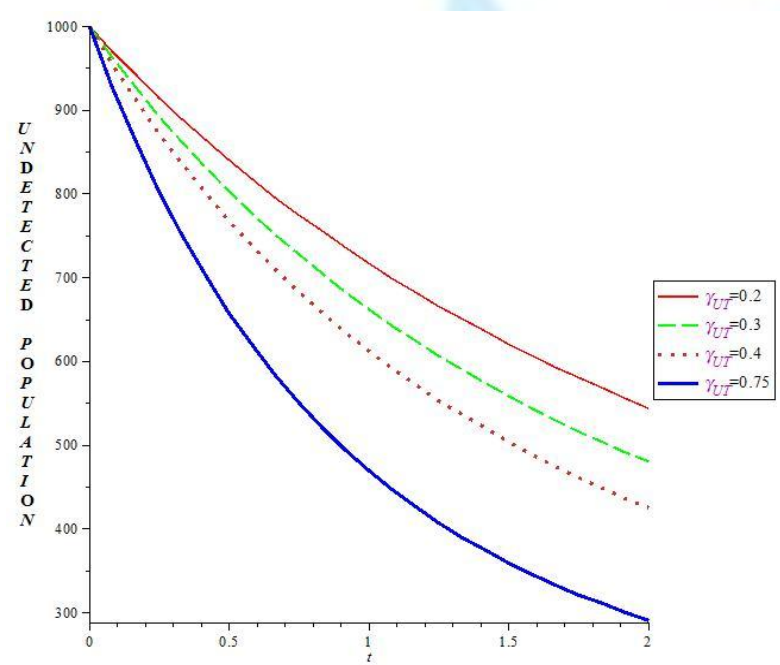

Fig. 7

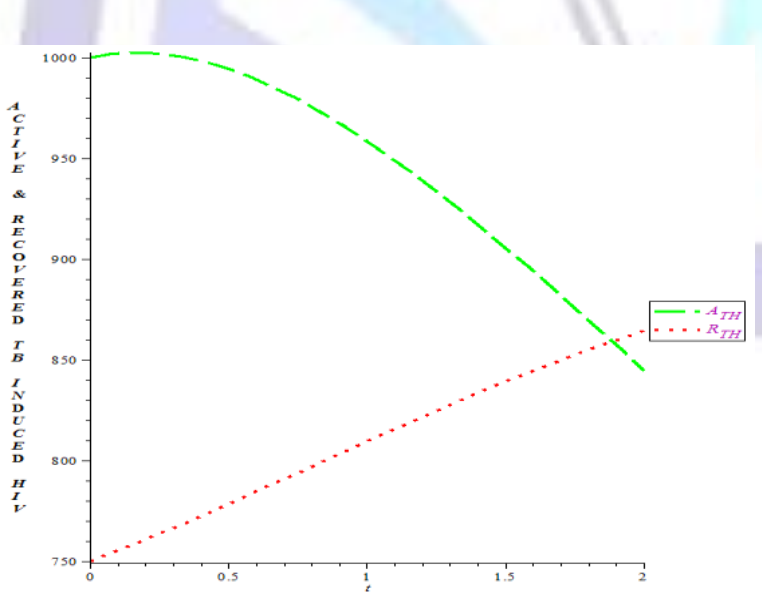

ISSN 2347-1921

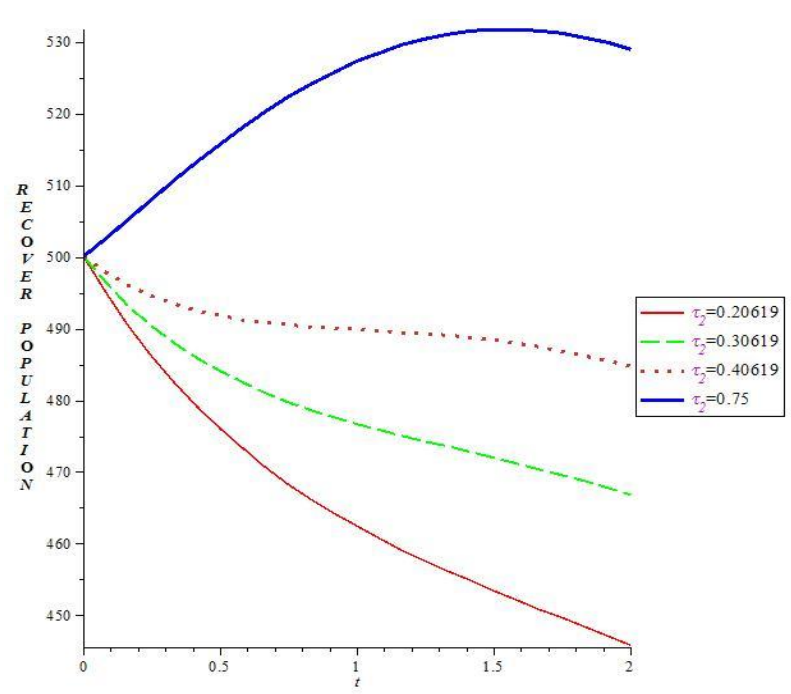

Fig. 6

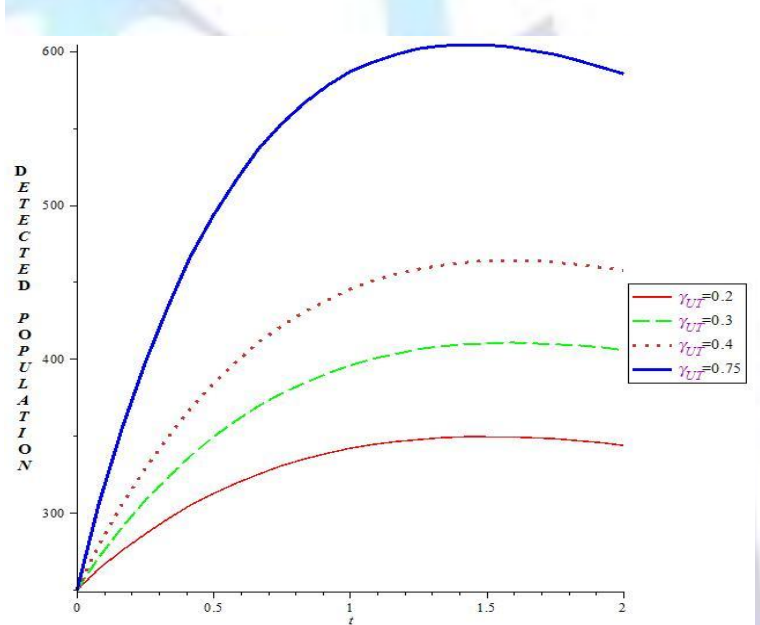

Fig. 8

Fig. 9 


\subsection{DISCUSSION}

Figure 1and 2 Show the effect of treatment on HIV infected detected and recovered individuals. It shows a decrease in the numbers of infected detected individuals and also increases the number of recovered individuals. It reduces infected detected individuals from 500 to 275 when the treatment rate $\tau_{1}$ is about $75 \%$ effective. Figure 3 and 4 has shown that detection rate has pronounced effect on HIV undetected infected and detected infected individuals. It shows that the higher the detection rate, the higher the infected detected individuals and the lower the undetected infected individuals. It reduces it to a minimal level when the detection rate $\gamma_{U H}$ is about 0.7 . Figure 5 and $\mathbf{6}$ Shown that treatment of TB has pronounced effect on TB infected detected individuals. It reduces the numbers of infected detected individuals and shows the increase in recovered individuals especially when the treatment rate $\tau_{2}$ is about $75 \%$ effective. Figure 7 and 8 Shown that the detection of TB infected undetected individuals reduces the spread of the disease in the community, it reduces the population of infected undetected individuals from 1000 to around 10 when the detection rate $\gamma_{U T}$ is about 0.7 . This suggests that, detection of infected undetected TB individuals plays a vital role in the control of TB disease.

Figure 9 Shows the pronounce effect of treatment on active TB induced HIV individuals, it reduces active TB induced HIV from 1000 to 840 individuals and increases recovered individuals due to the efficacy of the drugs.

\subsection{CONCLUSION}

This work deals with the formulation and analysis of mathematical model to have better understanding of detection of infected undetected individuals and treatment strategies on the dynamical spread of HIV-TB co-infection.

In this paper, a mathematical model showing the effect of detection rate of HIV, TB and HIV-TB co-infection has been analyzed separately. Both qualitative and numerical analysis of each model was carried out. Qualitative analysis of each model reveals well posedness and uniqueness of their solutions. Basic reproduction number $\left(R_{0}\right)$ was computed by next generation matrix as well as the stability of model equilibrium. It was established that each model has two equilibrium points, namely; disease free equilibrium which is locally asymptotically stable whenever $R_{0}<1$ and unstable endemic equilibrium whenever $R_{0}>1$.

In numerical analysis, detection of infected undetected individuals $\gamma_{U H}$ and $\gamma_{U T}$ plays a vital role in the control of HIV-TB co-infection; it was observed that the detection of undetected individuals reduced the spread of disease, the disease becomes more endemic due to the increment in the numbers of infected undetected individuals. It was also shown that TB fuels the progression of HIV into full blown AIDS in the absence of proper treatment, likewise HIV increases latent TB to active TB in the absence of treatment. The results shown that in the presence of treatment, the rate of Active TB and HIV decreases as the treatment increases and consequently, the recover TB and HIV individuals increase rapidly.

In conclusion, epidemiological features such as detection rate of infected undetected individuals and treatment rate of infected detected individuals play vital roles in the control of the spread of HIV- TB co-infection disease in the community. Medical practitioners and policy health makers need to increase the rate at which infected undetected individuals are detected for proper and timely treatment, also, drugs should be made available at a cheaper rate for the consumers.

\section{REFERENCES}

1. Adewale, S. O. Podder, C. N. and Gumel, A. B. (2009). Mathematical analysis of a Tuberculosis (TB) Transmission Model with DOTS. Canadian Applied Mathematics quarterly volume 17, number 1, spring 2009

2. Aparicio J.P., Capurro A.F. and Castillo-Chavez C. (2002) Markers of disease evolution. The case of Tuberculosis Theoretical Biology 215 , 227-237.

3. Bacaer N., Ouitki R., Pretorius C., Wood R. and Williams B., (2008) Modeling the joint epidemics of TB and HIV in a South African township, Journal of Mathematical Biology $57,557-593$.

4. Becerra M. C., Pachao-Torreblanca I. F., Bayona J., Celi R., Shin S. S., Kim J. Y., Farmer P. E. and Murray M. (2005). Expanding tuberculosis case detection by screening household contacts, Public Health Report. 120.

5. Brudney K. and Dobkin J.(1991) Resurgent T.B in New York City. HIV homelessness and the decline of TB control programs. Am Res. Respir Dis; 144; 745-749.

6. Castillo-Chavez C. and Song B.,(2004) Dynamical models of tuberculosis and their applications, Mathematical Biosciences 1, 361-404.

7. Chaisson R.E. and Martinson N.A. (2008) Tuberculosis on Africa combating an HIV driven crisis. N Eng J Med, 358.1089-1092.

8. Cohen T., Lipsitch M., Walensky R.P. and Murray M. (2006) Beneficial and perverse effects of Isoniazid preventive therapy for latent tuberculosis Infection in HIV-TB Co-infected populations, proceedings of the National Academy of Science of the United States of America 103 , 7042-7047

9. Colijn C., Cohen T. and Murray M. (2007). Emergent heterogeneity in declining tuberculosis epidemics. J. Theor. Biol. 247, 765-774. 
10. Daniel O. and Andrei K. (2007). Dynamics of tuberculosis: The effect of Direct Observation Therapy Strategy (DOTS) in Nigeria. Mathematical Modelling of Natural Phenomena. 2(1):101-113.

11. Derricks W.R. and Grossman S.I. (1976), Elementary Differential Equations with application. Addison Wesley Publications Company, Philippine.

12. Dye C., Garnett G. P., Sleeman K. and Williams B.G. (1998). Prospects for worldwide tuberculosis control under the WHO DOTS strategy. Direct observed short-course therapy. Lancet. 1998;352:1886-1891 doi:10.1016/S0140-6736(98)03199-7 pmid:

13. Fuzzi, et al. (1996) Journal of Biological mathematics, 37, p 371-375

14. Gumel A. and Song B. (2008). Existence of multiple stable equilibria for a multi-drug resistance model of mycobacterium TB. Mathematics Biosci. and Engineering S(3) 437-455.

15. Hausler H.P., Sinanovic E., Kumaranayake L., Naidoo P., Schoeman H. and Karpakis B. (2006) Costs of measures to control TB/HIV in public primary care facilities in Cape town S.A. Bulletin of the WHO 84, 528-536.

16. Health Protection Agency. (2007) TB in the UK: Annual report on TB Surveillance and control in the UK 2007. London: Health protection Agency centre for infections.

17. Hethcote H.W. and Thieme H.R. (1985). Stability of the endemic equilibrium in epidemic models with subpopulations. Math. Biosci. 167: 205-227.

18. Heymann D.L.( 2008) Control of communicable Disease Manual, American Public Health Association. Washington DC.

19. Huang W.,Cooke K., and Castillo-Chavez C. (1992).Stability and bifurcation for a multiple group model for the dynamics of HIV/AIDS transmission. SIAM J.Appl. Math. 52: 835-854.

20. Kirschner D.(1996). Using mathematics to understanding HIV immune dynamics. Notices of the AMS. 43: 191-202.

21. Lakshmikantham V., Leela S. and Martynyuk A.A. (1989). Stability Analysis of Nonlinear Systems. Marcel Dekker, Inc.,New York and Basel.

22. Marison L. (2001) The global epidemiology of HIV/AIDS, British Medical Bulletin 58, 7-18

23. Okuonghae D. and Korobeinikov A. (2007). Dynamics of tuberculosis: The effect of Direct Observation Therapy Strategy (DOTS) in Nigeria, Math. Modelling Natural Phenomena 2(1), 101-113.

24. Quinn T.C., (1996) Global burden of the HIV pandemic, Lancet 348, 99-106

25. Sanchez M.S., Lloyd-Smith J.O., Williams B.G., Porco T.C., Ryan S.J., Borgdorff M.W., Mansoer J., Dye C. and Getz W.M. (2009) Incongruent HIV and TB Co-dynamics in Kenya. Interacting epidemics monitor each other, Epidemics 1, 14-20.

26. Sharomi O., Podder C.N., Gumel A.B. and Song B. (2008). Mathematical analysis of the transmission dynamics of HIV/TB co-infection in the presence of treatment. Mathematical Biosciences and Engineering. 5(1):145-174.

27. Singer B.H. and Kirschner D.E. (2004) Influence of backward bifurcation on interpretation of Ro in a model of epidemic TB with re-infection. Mathematical Biosciences and Engineering 1(1) 81-93.

28. Smart T.,(2008) Think TB in people with HIV: The l's for TB control in people with HIV, Nam, London,

29. Smith P.G. and Moss A.R.,( 1994) Epidemiology of Tuberculosis in: Tuberculosis Pathogenic Protection and Control (B. R. Bloom, ed), ASM Press, Washington, 47-59.

30. Theurer C.P., Hopewell P.C., Etias D., Schecter G.F., Rutherford G.W. and Chaisson R.E., (1990) HIV infection in TB patients, Journal of infectious Diseases $162,8-12$.

31. Van den Driessche P. and Watmough J., (2002) Reproduction numbers and sub-threshold endemic equilibria for compartmental models of disease transmission, Mathematical Biosciences 180, 29-48.

32. Wang W. (2006). Backward bifurcation of an epidemic model with treatment.Math.Biosci.201 (1-2):5871.

33. West R.W. and Thompson J.R. (1996). Modelling the impact of HIV on the spread of tuberculosis in the United States. Mathematical Biosciences.143: 35-60.

34. Wigginton J.E. and Kirschner D.(2001). A model to predict cell mediated immune regulatory mechanisms during human infection with mycobacterium tuberculosis. J. Immunol.166(3):1951.

35. World Health Organization (WHO) Report 2010 Global Tuberculosis control (2010)

36. World Health Organization, Anti-tuberculosis drug resistance in the world, 2004, WHO/HTM/TB/2008.394.

37. World Health Organization, Global tuberculosis control: surveillance, planning, financing, 2008, WHO/HTM/TB/2008.393. Bibliography 83 